\title{
Visualization, characterization and modulation of calcium signaling during the development of slow muscle cells in intact zebrafish embryos
}

\author{
CHRIS Y. CHEUNG ${ }^{1}$ "\#, SARAH E. WEBB ${ }^{1}$, DONALD R. LOVE ${ }^{2}$ and ANDREW L. MILLER ${ }^{*}, 1,3$ \\ ${ }^{1}$ Division of Life Science and State Key Laboratory of Molecular Neuroscience, The Hong Kong University of \\ Science and Technology, Hong Kong, $P R C,{ }^{2}$ School of Biological Sciences, University of Auckland, \\ Auckland, New Zealand and ${ }^{3} \mathrm{MBL}$, Woods Hole, MA, USA
}

\begin{abstract}
Intact zebrafish embryos were used as an in vivo animal model to investigate the role of $\mathrm{Ca}^{2+}$ signaling during the differentiation of slow muscle cells (SMCs) within forming skeletal muscle. Transgenic zebrafish were generated using an $\alpha$-actin promoter that targeted apoaequorin expression specifically to muscle cells. Two distinct $\mathrm{Ca}^{2+}$ signaling periods (CSPs) were visualized in the developing SMCs: between $\sim 17.5-19.5$ hours post-fertilization (hpf) and after $\sim 23 \mathrm{hpf}$, separated by a $\sim 3.5 \mathrm{~h} \mathrm{Ca}^{2+}$ signaling quiet period. Further spatial characterization of these $\mathrm{Ca}^{2+}$ signals using confocal fluorescent microscopy and calcium green-1 dextran as a reporter, indicated that the earlier CSP displayed distinct nuclear and cytoplasmic components, whereas the later CSP was predominantly cytoplasmic. Both CSPs consisted of a series of oscillating $\mathrm{Ca}^{2+}$ waves generated at distinct frequencies, while the earlier CSP also displayed a slow rise then fall in the $\mathrm{Ca}^{2+}$ baseline-level. Imaging of cyclopamine- and forskolin-treated wild-type, or smo- mutant embryos, where SMCs do not form, confirmed the specific cell population generating the signals. Treating embryos with antagonists indicated that both $\mathrm{IP}_{3} \mathrm{Rs}$ and $\mathrm{RyRs}$ are responsible for generating the temporal characteristics of the $\mathrm{Ca}^{2+}$ signaling signature, and that the latter plays a necessary role in SMC differentiation and subsequent myotome patterning. Together, these data support and extend the proposition that specific spatiotemporal patterns of spontaneous $\mathrm{Ca}^{2+}$ signals might be used for different as well as combinatorial regulation of both nuclear and cytosolic signal transduction cascades, resulting in myofibrillogenesis in SMCs as well as myotome patterning.
\end{abstract}

KEY WORDS: $\mathrm{Ca}^{2+}$ imaging, $I P_{3} R, R y R$, slow muscle cell, transgenic zebrafish

\section{Introduction}

The regulation of intracellular $\mathrm{Ca}^{2+}$ concentration, $\left[\mathrm{Ca}^{2+}\right]_{\mathrm{i}}$, has been reported to play a critical role in many aspects of muscle development and function (Ferarri et al., 1996; Brennan et al., 2005; Campbell et al., 2006). While the majority of $\mathrm{Ca}^{2+}$-muscle studies has been conducted in vitro, with cells or explants in culture, relatively few have been conducted in vivo, i.e., using intact, normally developing animals. For example, using the C2C12 mouse muscle cell line, it has been shown that three different patterns of $\mathrm{Ca}^{2+}$ signals are generated during in vitro myogenesis (Lorenzon et al., 1997) and that the frequency of the
$\mathrm{Ca}^{2+}$ transients determines the time required for de novo sarcomere assembly (Fujita et al., 2007). In isolated chick skeletal myoblasts, David et al. (1981) showed that a net movement of $\mathrm{Ca}^{2+}$ is essential for myoblast fusion. Furthermore, in primary cultures of Xenopus embryonic myocytes, Ferrari et al. (1996)

\footnotetext{
Abbreviations used in this paper: CSP, calcium signaling period; DMSO, dimethyl sulphoxide; E-CC, excitation-contraction coupling; FMCs, fast muscle cells; hpf, hours post-fertilization; $\mathrm{IP}_{3}$ Rs, inositol 1,4,5-trisphosphate receptors; QP, calcium signaling quiet period; RLU, relative light units; RyRs, ryanodine receptors; SMCs, slow muscle cells; SP1, calcium signaling period 1 .
}

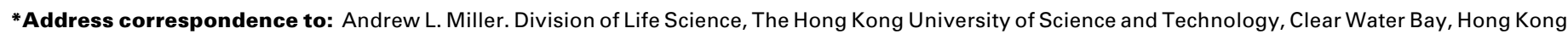
SAR, PRC. Fax: +852-2358-1559. e-mail: almiller@ust.hk - web: http://ihome.ust.hk/ aequorin/

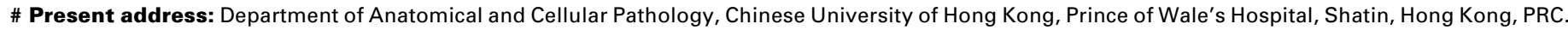

Supplementary Material (a movie and figure) for this paper is available at: http://dx.doi.org/10.1387/ijdb.103160cc 
reported that spontaneous $\mathrm{Ca}^{2+}$ signals are generated during just the first 15 hours in culture. These $\mathrm{Ca}^{2+}$ signals were shown to be generated by $\mathrm{Ca}^{2+}$ release from intracellular stores via ryanodine receptors (RyRs) and it was proposed that the signals play a role in the assembly of the myosin thick filaments (Ferrari et al., 1996; 1998). Ferrari and Spitzer, (1999) subsequently showed that similar $\mathrm{Ca}^{2+}$ dynamics also occur in the exposed myotome of dissected embryos and that these $\mathrm{Ca}^{2+}$ events seem to be correlated with somite formation and myocyte maturation. Li et al. (2004) then reported that spontaneous $\mathrm{Ca}^{2+}$ signals appear to regulate patterned actin assembly during myofibrillogenesis in cultured Xenopus myocytes, where blocking these signals disrupted the assembly of the actin thin filaments and the actinassociated z-disc affiliated proteins, titin and capZ. More recently, Brennan et al. (2005) reported that in intact zebrafish embryos, the release of intracellular $\mathrm{Ca}^{2+}$ from RyRs drives the initial contraction of slow muscle cells (SMCs) and that these contractions are responsible for the embryonic movements that occur at $\sim 17-22$ hpf. They also reported that this process is regulated by acetylcholine and results from spontaneous neuronal activity. Furthermore, blocking $\mathrm{Ca}^{2+}$ release with antagonists of either the acetylcholine receptor (AChR) or RyR disrupted the organization of the myofibrils without affecting the number of SMCs, or their elongation or migration. They thus proposed a critical role for nerve-mediated $\mathrm{Ca}^{2+}$ signals in the formation of physiologically functional slow muscle during zebrafish development (Brennan et al., 2005). Most recently, Campbell etal. (2006) reported ryanodine receptor (RyR)-mediated generation of both localized (i.e., in the subsarcolemma space), short duration $\mathrm{Ca}^{2+}$ transients (SDTs; lasting $\sim 2$ secs), as well as spontaneous global $\mathrm{Ca}^{2+}$ transients of long duration (LDTs; lasting $~ 80 \mathrm{sec}$ ) in embryonic myocytes prepared from neural plate (stage 15) Xenopus embryos. They proposed that the distinct spatiotemporal patterns of SDTs vs LDTs may be used for differential regulation of cytosolic signaling cascades, control of nuclear gene expression, and localized activation of assembly events at the sarcolemma (Campbell et al., 2006).

In this current study, we used intact zebrafish embryos as an in vivo animal model in order to extend our holistic understanding of the role of $\mathrm{Ca}^{2+}$ signaling during the differentiation of the SMCs of skeletal muscle. The various different types of skeletal muscle are all derived from the myotome region of the somites and their development is regulated by the interaction of a number of complex signaling networks (Weinberg et al., 1996; Brennan et al., 2002; Ochi and Westerfield, 2007). SMCs develop from a population of muscle precursor cells, termed adaxial cells, which lie adjacent to the notochord before segmentation begins (Devoto et al., 1996; Barresi et al., 2001). The adaxial cells are formed from the paraxial mesoderm when the notochord precursors secrete signaling molecules such as Hedgehog proteins (Currie

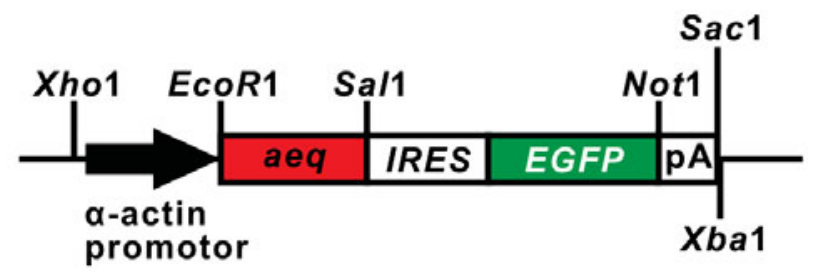

Fig. 1. Map of the p $\alpha-K S-a e q-I R E S-E G F P$ plasmid. and Ingham, 1996; Blagden et al., 1997; Stickney et al., 2000) following the convergence and extension cell movements that start in the earlier Gastrula Period (Kimmel et al., 1989; 1990). Shortly after somite formation, the adaxial cells, which are initially cuboidal in shape, elongate to extend across the anterior-posterior axis of the somite and then the majority migrate from the medial to lateral region of the somite to ultimately form a superficial monolayer of $\sim 20$ cells at the periphery of the myotome (Devoto et al., 1996; Du et al., 1997). Devoto et al. (1996) demonstrated that in the rostral trunk this process takes $\sim 5$ hours (i.e., from $\sim 17 \mathrm{hpf}$ to $\sim 23 \mathrm{hpf}$ or the 16- to 28-somite stages), during which time the adaxial cells differentiate into SMCs. The adaxial cells that do not migrate are called muscle pioneer cells; these remain in a medial location within the somite and form the horizontal myoseptum, which separates the dorsal and ventral regions of the myotome (Halpern et al., 1993). The non-adaxial cells in the myotome (called the lateral presomitic cells) also do not migrate and these differentiate into the fast muscle cells (FMCs; Devoto et al., 1996), via a process reported to be induced by the medial-to-lateral migration of the SMCs through the somite (Wolff et al., 2003; Henry and Amacher, 2004).

The first spontaneous contractions occur in the trunk muscle at $\sim 17$ hpf (i.e., 16-somite stage; Liu and Westerfield, 1992; SaintAmant and Drapeau, 1998; Drapeau et al., 2002), when the individual myotome blocks are innervated by axons of the caudal primary motoneurons (Eisen et al., 1986; Westerfield et al., 1986; Myers et al., 1986; Brennan et al., 2005). The spontaneous muscle contractions reach a peak frequency of $\sim 1 \mathrm{~Hz}$ by $\sim 19 \mathrm{hpf}$ and then decline again over the next few hours to reach a frequency of $\sim 0.1 \mathrm{~Hz}$ by $\sim 26 \mathrm{hpf}$ (Saint-Amant and Drapeau, 1998). These initial spontaneous muscle contractions are followed sequentially by touch-evoked rapid coils and organized swimming behavior, which start at $\sim 21 \mathrm{hpf}$ and $\sim 30 \mathrm{hpf}$, respectively (Saint-Amant and Drapeau, 1998).

In order to extend our understanding of the role of $\mathrm{Ca}^{2+}$ signaling during embryonic muscle development, complementary aequorin-based and fluorescence-based confocal $\mathrm{Ca}^{2+} \mathrm{im}$ aging techniques were applied. With regard to the former, we developed a new line of transgenic zebrafish that express apoaequorin specifically in the trunk musculature. Active aequorin in these transgenic zebrafish embryos was then reconstituted by incubation in a solution containing the apoaequorin co-factor, coelenterazine (Cheung et al., 2006). While aequorin-based imaging allows for the continuous, non-disturbing visualization of intact zebrafish embryos for long-duration developmental studies (for example, during the entire development of the embryonic trunk musculature), a drawback of this technique is that it only allows for two-dimensional and low resolution $\mathrm{Ca}^{2+}$ imaging. Thus, after we applied aequorin-based imaging to establish where and when $\mathrm{Ca}^{2+}$ transients arose in the developing musculature, we then examined the signals at higher spatial resolution using the fluorescent $\mathrm{Ca}^{2+}$ reporter, calcium green-1 dextran, in conjunction with confocal microscopy. The use of these two imaging techniques allowed us to add new spatial information, from both a rostrocaudal perspective, as well as at a sub-cellular level, to what is known about $\mathrm{Ca}^{2+}$ signaling in SMCs in an intact vertebrate system. In addition, it has allowed us to extend our understanding of the various $\mathrm{Ca}^{2+}$-release mechanisms responsible for generating the signals as well as to investigate (via their 
inhibition) the possible functional roles of these signals during SMC differentiation and myotome development.

\section{Results}

\section{Visualizing $\mathrm{Ca}^{2+}$ signals from transgenic fish that express apoaequorin in the musculature}

Transgenic zebrafish that express the apoaequorin (aeq) gene (Inouye et al., 1989) exclusively in the musculature were generated by microinjecting an aeq construct driven by an $\alpha$-actin promoter (Higashijima et al., 1997) into embryos at the 1-cell stage (Fig. 1). This plasmid also contained an EGFP marker gene, which was regulated by the IRES-sequence (a short sequence of DNA that initiates the internal translation of RNA) for the subsequent identification of transgenic fish. We have termed this $\alpha$-actin-apoaequorin-IRES-EGFP transgenic line of fish, $\alpha$-actinaeq. Use of the IRES-sequence enables the translation of both aeq and EGFP from a single mRNA, thus the expression level and distribution of EGFP reflects the expression level and distribution of apoaequorin (Jang et al., 1988; Fahrenkrug et al., 1999; Wang et al., 2000).

Using this plasmid, a single transgenic founder fish $\left(F_{0}\right)$ was identified from 85 candidate fish. This $F_{0}$ fish was then out-bred with a wild-type fish to establish the $F_{1}$ transgenic line. The $F_{1}$ embryos that expressed EGFP (Fig. 2A) were identified and raised to adults for the generation of a stable homozygous $F_{2}$ line. In this transgenic line, the EGFP and thus the apoaequorin were expressed at low levels at $\sim 12$ hpf (i.e., 6-somite stage; Fig. 2B) and the level of expression increased in an approximately linear manner over the time range measured, i.e., up to $24 \mathrm{hpf}$. Fig. 2C shows a transverse cross section through the myotome of a representative $(n=5)$ transgenic zebrafish embryo at $\sim 24 \mathrm{hpf}$ and shows that the EGFP (Fig. 2Ci) and thus apoaequorin were expressed throughout the entire musculature including the slow muscles (the myosin of which was labeled with the F59 antibody; Fig. 2Cii and 2Ciii). Together, these results suggest that this $\alpha-$ actin-aeq fish line can be used to visualize and characterize the $\mathrm{Ca}^{2+}$ signals generated in the developing trunk musculature (Fig. 2D) between approximately $16 \mathrm{hpf}$ and $25 \mathrm{hpf}$, (i.e., the period reported in this study).

\section{Characterizing the trunk $\mathrm{Ca}^{2+}$ signals in zebrafish with aequorin-based $\mathrm{Ca}^{2+}$ imaging}

Transgenic embryos $(n=6)$ were incubated in $f$-coelenterazine solution to reconstitute active aequorin, and the $\mathrm{Ca}^{2+}$ signals generated in the developing trunk between $\sim 17 \mathrm{hpf}$ to $\sim 24.5 \mathrm{hpf}$ were visualized using our PIMS (Fig. 3Ai). Using a region of interest (ROI) that covered the entire embryo (Fig. 3Aii), and acquiring the luminescent data in photons/sec, two distinct periods of $\mathrm{Ca}^{2+}$ signaling were observed in the developing anterior trunk (Fig. 3Ai). The first period of $\mathrm{Ca}^{2+}$ signaling was comprised of two main components: The first to appear was rapid $\mathrm{Ca}^{2+}$ spiking, which began suddenly at $17.5 \mathrm{hpf}$ (i.e., the 17-somite stage), where the maximum amplitude of signaling was detected almost immediately. Spiking activity lasted until $19.5 \mathrm{hpf}$ (i.e., the 21-somite stage). The spiking frequency was not, however,

Fig. 2. Characterization of the transgenic zebrafish line that expresses the apoaequorin and EGFP genes, which are targeted to the skeletal muscle via a muscle specific $\alpha$-actin promoter. (A) Expression of EGFP (see arrowhead) in the trunk musculature of a representative $\alpha$-actin-aeq transgenic $\left(F_{1}\right)$ embryo at 26 hpf. This embryo was bred by crossing the single p $\alpha$-KS-Aeq-IRES-EGFP plasmid-injected founder fish $\left(F_{0}\right)$ generated, with a wild-type fish. Ant. and Pos. are anterior and posterior, respectively. (B) Profile of apoaequorin expression in the $F_{1}$ transgenic embryos from 12 hpf to 24 hpf. (Bi) Representative example ( $n=3)$ of a Western blot and (Bii) a line graph (mean \pm SEM; $n \geq 3$ ) to illustrate the relative level and temporal expression profile of apoaequorin in extract prepared from the transgenic embryos. Aeq, aequorin control; WT, wild-type embryos. Extract prepared from 3 embryos was loaded into each lane. (C) Cross section of the myotome of a representative $(n=5)$ transgenic zebrafish embryo at 24 hpf illustrating (Ci) the expression of EGFP, (Cii) the slow muscles (labeled via immunohistochemis-

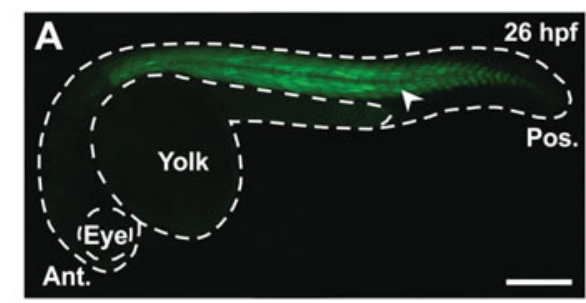

\section{Bi}
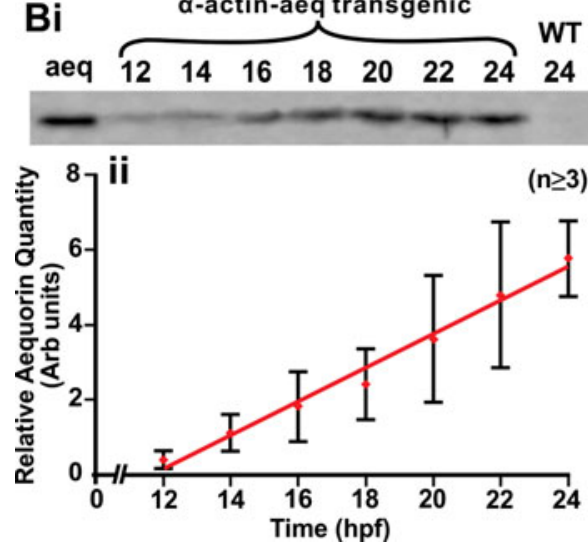
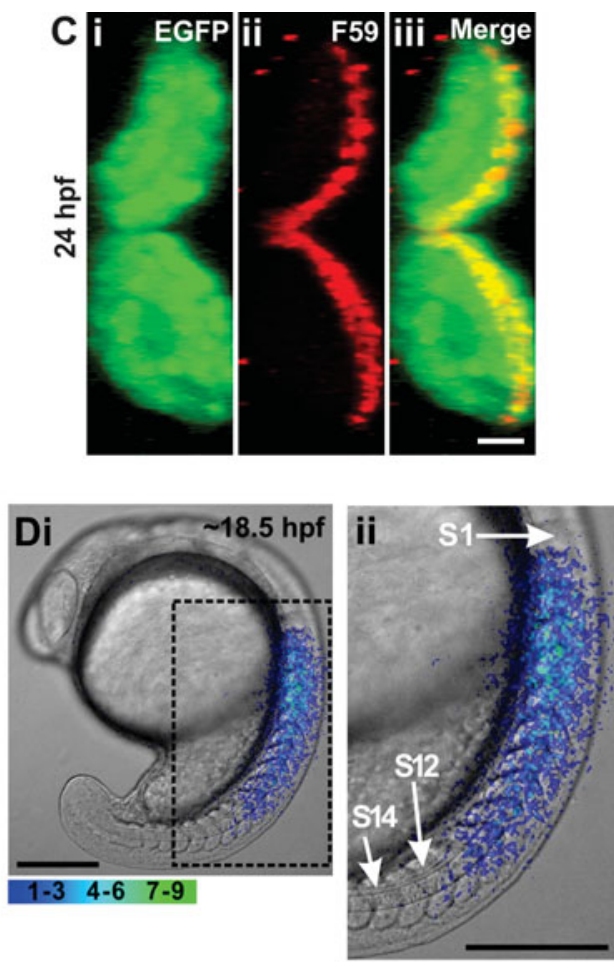

try with the F59 myosin heavy chain antibody), and (Ciii) the EGFP and F59-labeled images when merged, such that the yellow color indicates the region of overlap. (Di) A representative example $(n=4)$ of the spatial pattern of $\mathrm{Ca}^{2+}$ transients generated in the trunk of an apoaequorin expressing transgenic embryo at $\sim 18.5 \mathrm{hpf}$. This image represents $10 \mathrm{sec}$ of accumulated light superimposed on to the appropriate bright-field image. The region of the embryo bounded by the black dashed square is shown at higher magnification in Dii. S1, S12 and S14 are somites 1, 12 and 14, respectively. Color scale indicates luminescent flux in photons/pixel. Scale bars are (A) $250 \mu \mathrm{m},(C) 10 \mu \mathrm{m}$, and (D) $200 \mu \mathrm{m}$. 
constant during this time, but altered in a distinct and highly reproducible manner. Unlike the sudden initiation of the spiking activity, its termination was the result of a steady decline in spiking amplitude (Fig. 3Ai). The second main component of the $\mathrm{Ca}^{2+}$ signaling activity was related to the baseline level of the $\mathrm{Ca}^{2+}$ spiking. This did not remain constant during the spiking period, but displayed a gradual increase, which started at $\sim 18 \mathrm{hpf}$, reached a maximum at $\sim 18.75 \mathrm{hpf}$ and then returned to its original resting value by $\sim 19.5 \mathrm{hpf}$. This was concomitant with the cessation of the $\mathrm{Ca}^{2+}$ spiking activity. Thus, from $\sim 18$ hpf onwards, the $\mathrm{Ca}^{2+}$ spiking activity was superimposed on top of this baseline modulation. We have termed this second $\mathrm{Ca}^{2+}$ signaling feature the "slow rising component". We have called the combination of $\mathrm{Ca}^{2+}$ spiking and the slow rising component, " $\mathrm{Ca}^{2+}$ Signaling Period 1" (or SP1).
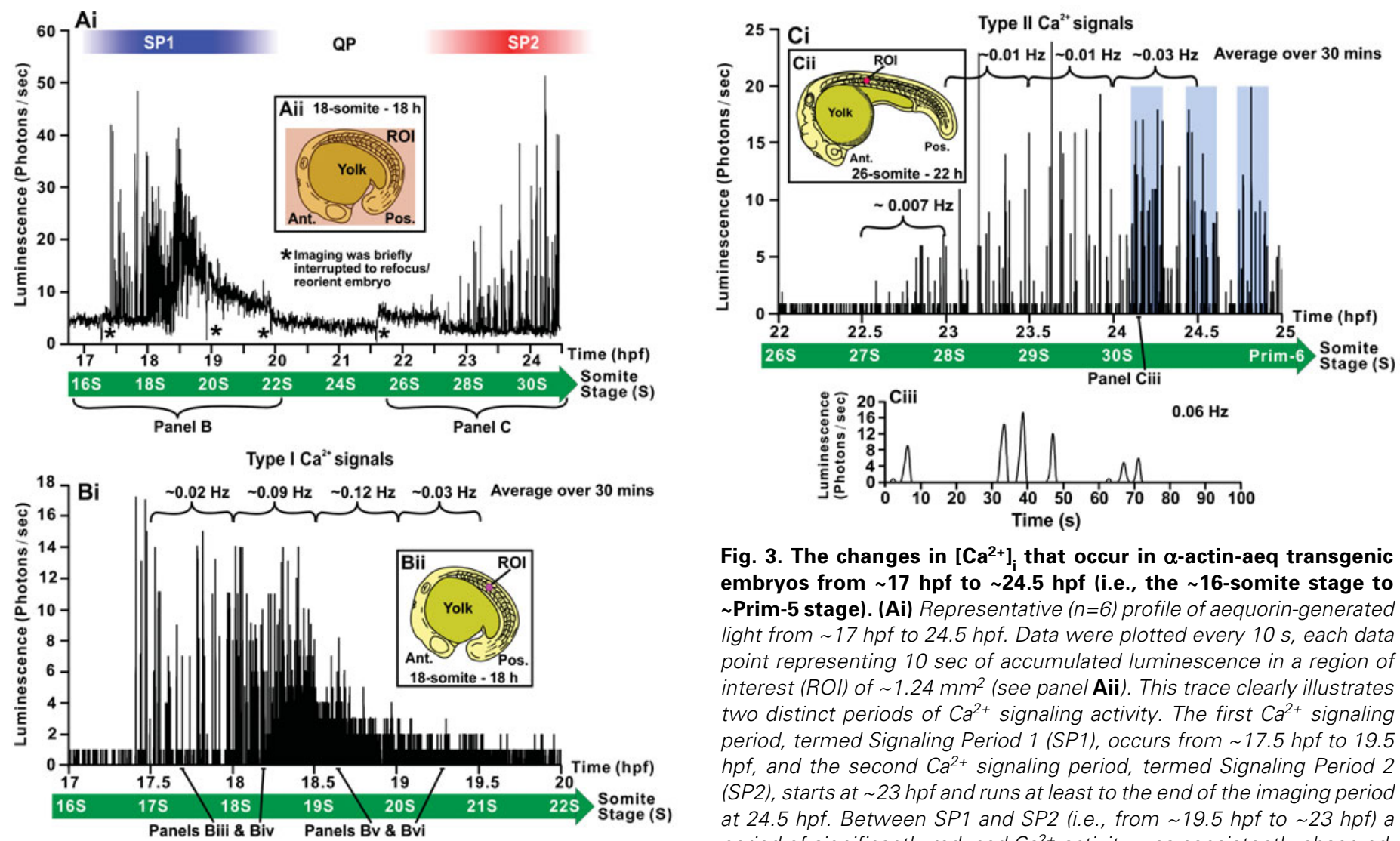

Fig. 3. The changes in $\left[\mathrm{Ca}^{2+}\right]_{\mathrm{i}}$ that occur in $\alpha$-actin-aeq transgenic embryos from $\sim 17 \mathrm{hpf}$ to $\sim 24.5 \mathrm{hpf}$ (i.e., the $\sim 16$-somite stage to $\sim$ Prim-5 stage). (Ai) Representative $(n=6)$ profile of aequorin-generated light from $\sim 17 \mathrm{hpf}$ to $24.5 \mathrm{hpf}$. Data were plotted every $10 \mathrm{~s}$, each data point representing $10 \mathrm{sec}$ of accumulated luminescence in a region of interest (ROl) of $\sim 1.24 \mathrm{~mm}^{2}$ (see panel Aii). This trace clearly illustrates two distinct periods of $\mathrm{Ca}^{2+}$ signaling activity. The first $\mathrm{Ca}^{2+}$ signaling period, termed Signaling Period 1 (SP1), occurs from $17.5 \mathrm{hpf}$ to 19.5 hpf, and the second $\mathrm{Ca}^{2+}$ signaling period, termed Signaling Period 2 (SP2), starts at $\sim 23 \mathrm{hpf}$ and runs at least to the end of the imaging period at 24.5 hpf. Between SP1 and SP2 (i.e., from 19.5 hpf to 23 hpf) a period of significantly reduced $\mathrm{Ca}^{2+}$ activity was consistently observed. We have termed this the $\mathrm{Ca}^{2+}$ signaling quiet period (QP). (Bi) Profile of the SP1 $\mathrm{Ca}^{2+}$ signals

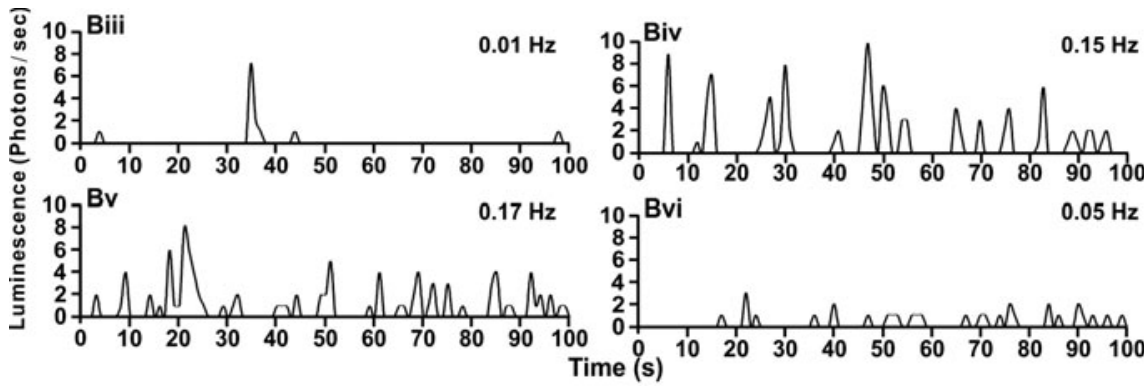
alone (i.e., from $17 \mathrm{hpf}$ to $20 \mathrm{hpf}$ ). (Biii, Biv, Bv, Bvi) Profiles of the $\mathrm{Ca}^{2+}$ signals generated over a period of $100 \mathrm{sec}$ from 17.5-18 hpf, 18-18.5 hpf, 18.5-19 hpf and 19-19.5 hpf, respectively, as indicated in panel (Bi). (Ci) Profile of the $\mathrm{Ca}^{2+}$ signals generated at the end of the QP and start of SP2 (i.e., from $22 \mathrm{hpf}$ to $25 \mathrm{hpf}$ ). The shaded regions show bursts of $\mathrm{Ca}^{2+}$ signaling activity. (Ciii) Profile of the $\mathrm{Ca}^{2+}$ signals generated over a period of 100 sec from 24-24.5 hpf, as shown in panel (Ci). For the profiles in $B$ and $C$, data were plotted every 1

s, each data point representing 1 sec of accumulated luminescence for ROls located in somite 8 (i.e., $~ 5000 \mu$
The average frequency (in $\mathrm{Hz}$ ) of the $\mathrm{Ca}^{2+}$ signals generated in 30-min periods are shown in panels (Bi, Ci). 
to somite 8 was used (Fig. 3Bii and $3 \mathrm{Cii}$ ), and the data were reviewed at higher temporal resolution. For both signaling periods, we made the assumption that rates of luminescence of 2 photons/sec and above were "real" $\mathrm{Ca}^{2+}$ signals and anything below this was background noise. During SP1, the frequency of signal generation showed distinct variations (Fig. 3Bi). The lowest frequency of signaling $(\sim 0.02 \mathrm{~Hz})$ occurred during the first $\sim 30$ minutes of SP1 (i.e., between $17.5 \mathrm{hpf}$ and $18 \mathrm{hpf}$ ). The frequency of the signaling then increased $\sim 4$-fold to $\sim 0.09 \mathrm{~Hz}$ between $18 \mathrm{hpf}$ and $18.5 \mathrm{hpf}$, and then peaked at $\sim 0.12 \mathrm{~Hz}$ between $18.5 \mathrm{hpf}$ and $19 \mathrm{hpf}$. Between $19 \mathrm{hpf}$ and $19.5 \mathrm{hpf}$, the frequency of signals then dropped again to $\sim 0.03 \mathrm{~Hz}$, and finally fell to zero, indicating the beginning of the QP. The varying frequencies observed during SP1 are also shown in the higher temporal resolution plots (Fig. 3Biii-3Bvi) and were a conserved feature seen in all embryos examined (Fig. 4A). When signaling resumed at the start of SP2, the frequency of the $\mathrm{Ca}^{2+}$ signals was initially just $\sim 0.007 \mathrm{~Hz}$ and then as the SP2 signals became established at $\sim 24 \mathrm{hpf}$, the frequency increased to $\sim 0.03 \mathrm{~Hz}$ (Fig. 3Ci, Ciii). Between 24-24.5 hpf the SP2 $\mathrm{Ca}^{2+}$ transients were also characterized by $\sim 12-\mathrm{min}$ bursts of activity followed by $\sim 5$-min periods of relative inactivity (see shaded regions in Fig. 3Ci).

What we have called SP1 and SP2 in the $\alpha$-actin-aeq transgenic embryos, we previously described as "two distinct periods of $\mathrm{Ca}^{2+}$ signaling" in zebrafish embryos that were injected with apoaequorin-mRNA (aeq-mRNA) to express aequorin transiently in the entire embryo (described by Cheung et al., 2006). We conducted a detailed comparison of SP1 and SP2 $\mathrm{Ca}^{2+}$ signals during restricted time windows using both $\alpha$-actin-aeq transgenic embryos and aeq-mRNA injected embryos ( $n \geq 3$ for each; see Fig. $4 \mathrm{~A}, \mathrm{~B})$. All statistical analyses were conducted using the student's $t$-test for unpaired samples.

Fig. 4A shows that the frequencies of the SP1 $\mathrm{Ca}^{2+}$ signals (i.e., between 17.5-18 hpf, 18-18.5 hpf, 18.5-19 hpf and 19-19.5 hpf) generated in the $\alpha$-actin-aeq transgenic embryos were not statistically different from those generated in the aeq-mRNA injected embryos during the respective time periods. In SP1, the frequency of the $\mathrm{Ca}^{2+}$ signals was significantly lower (at $\mathrm{p}<0.01$ ) between 17.5-18 hpf (i.e., $~ 0.02 \mathrm{~Hz}$ ) than at any other time in SP1 and SP2. The frequency increased from $\sim 0.02$ between 17.5-18 hpf to $\sim 0.09 \mathrm{~Hz}$ between 18-18.5 hpf, and reached a maximum of $\sim 0.13 \mathrm{~Hz}$ between 18.5-19 hpf. The frequency of the signals then decreased to $\sim 0.06 \mathrm{~Hz}$ between 19-19.5 hpf. The frequency of the SP2 $\mathrm{Ca}^{2+}$ signals (i.e., $\sim 0.03 \mathrm{~Hz}$ ) was significantly lower than that generated between 18-18.5 hpf (i.e., $\sim 0.09 \mathrm{~Hz}$ ) and between 18.5-19 hpf (i.e., $\sim 0.13 \mathrm{~Hz}$ ), at $\mathrm{p}<0.01$. On the other hand, the frequency of the SP2 signals was not statistically different from the frequency of the SP1 signals at 19-19.5 hpf (i.e., $\sim 0.06 \mathrm{~Hz}$ ).

The duration of the SP1 and SP2 $\mathrm{Ca}^{2+}$ signals for each of the $\alpha$-actin-aeq transgenic and aeq-mRNA injected embryos was also compared (Fig. 4B). We followed the protocol described by Brennan et al. (2005), and calculated the duration as the time taken for a signal to decay from the maximum amplitude to half of that value. The durations that were calculated for each of the four periods comprising SP1 (i.e., between 17.5-18 hpf, 18-18.5 hpf, 18.5-19 hpf and 19-19.5 hpf) generated in the $\alpha$-actin-aeq transgenic embryos were not significantly different from those generated in the aeq-mRNA injected embryos, during the respective time periods. In SP1, the $\mathrm{Ca}^{2+}$ signals between 17.5-18 hpf had the longest duration (i.e., $0.84 \mathrm{sec}$ ). The duration of the signals then decreased through SP1 such that by 19-19.5 hpf, the mean duration of the signals was just $\sim 0.46 \mathrm{sec}$. The duration of the signals between 18-18.5 hpf was significantly lower than those between $17.5-18 \mathrm{hpf}$ at $p<0.01$. Likewise, the duration of the signals between 19-19.5 hpf was significantly lower

Fig. 4. Characteristics of the SP1 and SP2 $\mathrm{Ca}^{2+}$ signals generated in somites 8 of representative $\alpha$-actin-aeq transgenic embryos and aeq-mRNA injected embryos. The vertical bar charts show the (A) frequency and (B) duration of the $\mathrm{Ca}^{2+}$ signals at 17.5-18 hpf, 18-18.5 hpf, 18.5-19 hpf, 19-19.5 hpf and 24-24.5 hpf. Data are expressed as means \pm SEMs of 3-4 independent experiments. (C,D) Horizontal bar charts indicating the location with respect to formed somites of the trunk $\mathrm{Ca}^{2+}$ signals generated in 4 representative apoaequorin transgenic embryos (E1 to E4) and in 4 representative aeqmRNA injected embryos (E5 to E8) at (C) $~ 17.5 \mathrm{hpf}$ and (D) $\sim 18.5$ hpf. (E) Schematics of an embryo at $\sim 18$ hpf to show the somites, numbered 1-18. 
than those between $18.5-19 \mathrm{hpf}$ at $\mathrm{p}<0.01$. On the other hand, all the signals between 18-18.5 hpf and 18.5-19 hpf had a similar duration of $\sim 0.7 \mathrm{sec}$. The durations of the SP2 $\mathrm{Ca}^{2+}$ transients $(\sim 0.6 \mathrm{sec})$ were significantly greater than those of the $19-19.5 \mathrm{hpf}$ SP1 signals (at $\mathrm{p}<0.01$ ).

Fig. $4 \mathrm{C}, \mathrm{D}$ are horizontal bar charts to show the location of the $\mathrm{SP} 1 \mathrm{Ca}^{2+}$ signals with respect to the formed somites in $4 \alpha$-actinaeq transgenic embryos and 4 aeq-mRNA injected embryos. In all 8 examples, these signals were generated in a discrete number of somites in the anterior trunk. At the start of the SP1 $\mathrm{Ca}^{2+}$ signaling window (i.e., $17.5 \mathrm{hpf}$; the 17-somite stage), the low frequency (i.e., $\sim 0.02 \mathrm{~Hz}$ ) $\mathrm{Ca}^{2+}$ signals were generated between somites 4 and 10 in all 8 embryos examined at this stage of development (Fig. 4C). At $\sim 18.5 \mathrm{hpf}$ (i.e., the 19 somite stage), when the frequency of the $\mathrm{Ca}^{2+}$ signals rose to $\sim 0.12 \mathrm{~Hz}$, these signals were generated between somites 2 and 12, and in this case were never seen to extend into somite 1 in an anterior direction, or beyond somite 12 in a posterior direction (Fig. 4D). At both time points analyzed (i.e., $17.5 \mathrm{hpf}$ and $\sim 18.5 \mathrm{hpf}$ ), there was no difference in the anterior-to-posterior spread of the signals generated, when comparing the transgenic embryos with the mRNA-injected embryos (Fig. 4 C,D).

\section{Further characterization of the trunk $\mathrm{Ca}^{2+}$ signals with cal-} cium green-1 dextran and confocal imaging

The fluorescent $\mathrm{Ca}^{2+}$ reporter, calcium green-1 dextran (10S) in conjunction with confocal microscopy, was used to confirm and support the data collected using aequorin-based imaging. In addition, the higher spatial resolution of this imaging technique enabled us to characterize the spatial aspects of the trunk $\mathrm{Ca}^{2+}$ signals in greater detail.

Fig. 5 shows a representative example $(n=3)$ of the $\mathrm{Ca}^{2+}$ signals generated in individual muscle cells in somite 8 at 17.5 hpf (i.e., the 17-somite stage). Fig. 5A shows an example of two prominent $\mathrm{Ca}^{2+}$ signals that were visualized from two individual muscle cells located in somite 8 , both of which lasted for at least $6 \mathrm{sec}$. These $\mathrm{Ca}^{2+}$ signals were generated predominantly in the nucleus and peri-nuclear region of each individual muscle cell, but were also seen to spread into the cytoplasmic region of each cell. This is especially obvious in the lower of the two cells shown in this example (see arrowheads in Fig. 5 AiiAiv). The profiles of two consecutive $\mathrm{Ca}^{2+}$ signals generated in the upper of the two muscle cells in somite 8 are shown in Fig. $5 \mathrm{Bi}$, and these again indicate that the total duration of the $\mathrm{Ca}^{2+}$ signals is in the range of 5 to 10 seconds. The reliability of the calcium green-1 dextran signals was confirmed via the co-injection of and subsequent imaging with an equivalent molecular weight molecule, rhodamine B dextran (Fig. 5Bii). The $\mathrm{Ca}^{2+}$ signals shown in Fig. 5 correlated with a spontaneous contraction in the muscle cells (see Supplemental Movie \#1).

Fig. 6 is a representative example $(n=3)$ of the $\mathrm{Ca}^{2+}$ signals generated by individual muscle cells in somites $7-10$ at $\sim 18.5 \mathrm{hpf}$ (i.e., the 19-somite stage). Fig. $6 \mathrm{~A}$ shows a representative $\mathrm{Ca}^{2+}$ signal that was imaged in the trunk, which lasted $\sim 5 \mathrm{sec}$. The profiles of the $\mathrm{Ca}^{2+}$ signals generated over a period of $100 \mathrm{sec}$ in somites 7-10 (Fig. 6 Bi-Biv) show that the signals generated in one somite were in phase with those generated in the other somites (indicated by the red dashed lines). In addition, the $\mathrm{Ca}^{2+}$ signals generated in the dorsal, medial and ventral parts of a somite (i.e., somite 9; Fig. 6C) were also found to be in synchrony. Again, the reliability of the calcium green-1 dextran signals was confirmed via the co-injection and subsequent imaging of rhodamine $B$ dextran (Fig. 6Bv). Moreover, the $\mathrm{Ca}^{2+}$ signals observed at $\sim 17.5$ hpf and $\sim 18.5$ hpf via confocal microscopy support and confirm the SP1 $\mathrm{Ca}^{2+}$ signals generated in the $\alpha$-actin-aeq transgenic fish at the same time (compare Fig. 5Bi with Fig. 3Biii and Fig. 6Bi-Biv with Fig. 3Bv).

Using calcium green-1 dextran and confocal microscopy, we also investigated $\mathrm{Ca}^{2+}$ signals at the level of a single muscle cell (Fig. 7). Fig. $7 \mathrm{C}, \mathrm{D}$ show a representative example of a $\mathrm{Ca}^{2+}$

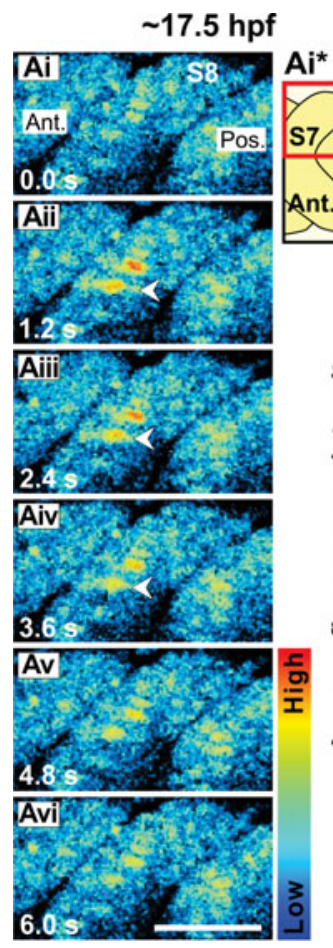

$\mathrm{Ai}^{*}$
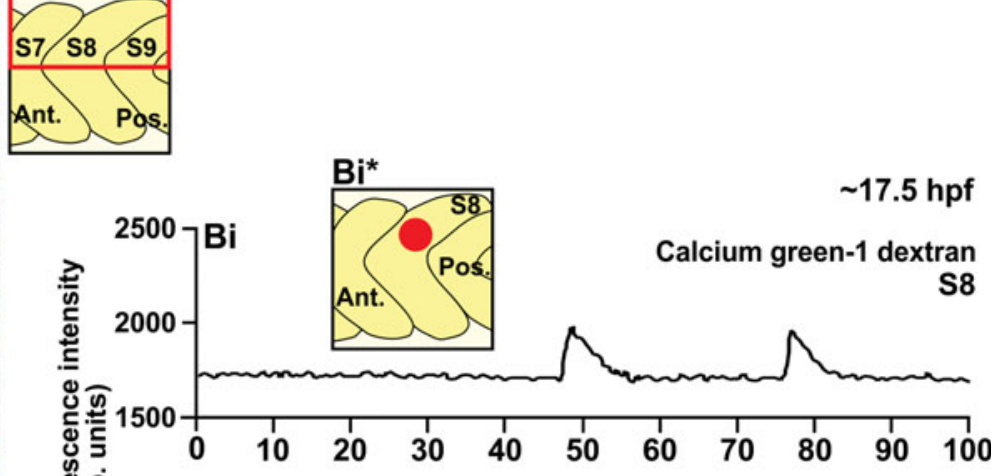

2500 Bii

Rhodamine B dextran

S8

$2000-$

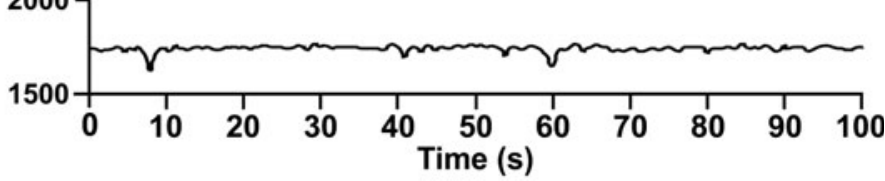

Fig. 5. An example of the $\mathrm{Ca}^{2+}$ signals generated in the trunk of a wild-type embryo at $\sim 17.5 \mathrm{hpf}$ (i.e., the 17-somite stage), as visualized by confocal microscopy using calcium green-1 dextran. (Ai-Avi) Representative ( $n=3$ ) single confocal sections to show the $\mathrm{Ca}^{2+}$ signals generated in the dorsal half of somite 7, 8 and 9 (see the schematic, panel $A i^{*}$ ). The time interval between each image was $\sim 1.2$ sec. The color scale represents the level of $\left[\mathrm{Ca}^{2+}\right]_{i}$, where red indicates a high level and blue indicates a low level. Ant. and Pos. are anterior and posterior, respectively. Scale bar, $50 \mu \mathrm{m}$. (Bi) Temporal profile of the average calcium green-1 dextran fluorescence intensity (in Arb. units) recorded in an ROI covering 4-5 SMCs (i.e., $~ 800 \mu^{2}$ ) placed in the dorsal part of somite 8 of this wild-type embryo at $\sim 17.5 \mathrm{hpf}$ (see schematic, panel $B i^{*}$ ). (Bii) Temporal profile of the average rhodamine $B$ dextran fluorescence intensity (in Arb. units) recorded in the same ROI over the same time period. 
signal generated in a single cell in somite 10 (Fig. 7B) at $\sim 18.5$ hpf. In this case, the $\mathrm{Ca}^{2+}$ signal was generated in the nucleus first, after which it spread through the cytoplasm. In addition, the nuclear $\mathrm{Ca}^{2+}$ signal remained relatively high even when the $\mathrm{Ca}^{2+}$ signal in the cytoplasm had decreased to the resting level again.

Fig. 8 shows a representative example $(n=3)$ of an SP2 $\mathrm{Ca}^{2+}$ signal generated in the trunk at $\sim 24 \mathrm{hpf}$ (i.e., the Prim-5 stage). Fig. 8A shows a representative $\mathrm{Ca}^{2+}$ signal that was imaged in the ventral portion of somite 8 , which lasted for $\sim 1.4 \mathrm{sec}$. The $\mathrm{Ca}^{2+}$ signal was generated in the entire cell when the muscle contracted. However, unlike the $\mathrm{Ca}^{2+}$ signals generated at $\sim 17.5$ hpf and $\sim 18.5 \mathrm{hpf}$, where the signal was generated mainly in the nucleus, at $\sim 24 \mathrm{hpf}$ the $\mathrm{Ca}^{2+}$ signals appeared to be mainly cytosolic. The profiles of the $\mathrm{Ca}^{2+}$ signals and the rhodamine- $\mathrm{B}$ dextran generated fluorescence recorded over a period of 100 $\mathrm{sec}$ in somite 8 are shown in $\mathrm{Fig} .8 \mathrm{Bi}$ and $8 \mathrm{Bii}$, respectively.

\section{Effect of cyclopamine and forskolin on slow muscle devel- opment and $\mathrm{Ca}^{2+}$ signaling in the trunk}

During confocal imaging, the cells that generated the SP1 $\mathrm{Ca}^{2+}$ signals appeared, from their morphology and location within the somite, to be SMCs (see Figs. 5 and 6). To confirm whether this was the case, $\alpha$-actin-aeq transgenic embryos were treated with either $0.1 \mathrm{mM}$ cyclopamine (Chen et al., 2002) or $0.15 \mathrm{mM}$ forskolin (Barresi et al., 2000). It has previously been reported that these drugs inhibit the development of SMCs by blocking different aspects of sonic hedgehog signaling. Cyclopamine antagonizes the function of the smoothened protein, which is a

Fig. 6. An example of the $\mathrm{Ca}^{2+}$ signals generated in the trunk of a wild-type embryo at $\sim 18.5 \mathrm{hpf}$ as visualized by confocal microscopy using calcium green-1 dextran. (Ai-Av) Representative $(n=3)$ single confocal sections to show the $\mathrm{Ca}^{2+}$ signals generated in somites 7, 8, 9 and 10 at 18.5 hpf (i.e., the 19-somite stage). The time interval between each image was $1.16 \mathrm{sec}$. The color scale represents the level of $\left[\mathrm{Ca}^{2+}\right]_{i}$, where red indicates a high level and blue indicates a low level. Ant. and Pos. are anterior and posterior, respectively. Scale bar is $50 \mu \mathrm{m}$. (BiBiv) Temporal profiles of average calcium green-1 dextran fluorescence intensity (in Arb. units) recorded in four ROls leach covering 4-5 SMCs, i.e., $\left.\sim 800 \mu \mathrm{m}^{2}\right)$ placed in the middle of somites 7, 8, 9 and 10 of this wild-type embryo at $18.5 \mathrm{hpf}$ (see schematic, panel $\left.B i^{*}\right)$. The three red dashed lines help to illustrate the synchronized nature of these signals. (Bv) Temporal profile of average rhodamine B dextran fluorescence intensity (in Arb. units) recorded in an ROI placed in the middle of somite 8 (see schematic, panel $\left.B v^{*}\right)$. (C) Temporal profiles of average calcium green-1 dextran fluorescence intensity (in Arb. units) in the dorsal (green), medial (red) and ventral (blue) regions of somite 9 , which indicate that the majority of the $\mathrm{Ca}^{2+}$ signals in the dorsal, medial and ventral regions of the somite also occur in synchrony. (C*) Schematic to show the dorsal (D), medial (M) and ventral (V) ROIs. component of the hedgehog signaling pathway (Chen et al., 2002), while forskolin indirectly inhibits the response of cells to hedgehog signaling by activating CAMP-dependent protein kinase (PKA; Barresi et al., 2000). Embryos treated with these drugs exhibited several gross developmental abnormalities at 24 hpf, compared with the untreated and DMSO controls (Fig. $9 \mathrm{Ai}-$ Di). Both drugs induced abnormalities in the trunk and tail, and forskolin additionally caused abnormal head formation (compare Fig. 9 Ci, Di with Fig. 9 Ai, Bi). Fig. 9Aii-Dii are bright-field images of the trunk at higher magnification. While the somites in the untreated and DMSO-treated embryos displayed a normal chevron-shape, those in the embryos treated with cyclopamine or forskolin were U-shaped (compare Fig. 9 Cii, Dii with Fig. 9 Aii, Bii). In addition, immunohistochemistry of the slow muscle fibers with the F59 antibody shows that cyclopamine and forskolin treatment almost completely blocked slow muscle formation at 24 hpf (compare Fig. 9 Ciii, Diii with Fig. 9 Aiii, Biii). Fig. 9 Aiv-Div show the profiles of aequorin-generated light from transgenic embryos that were either untreated (Fig. 9Aiv; $n=9$ ), or else were treated with DMSO (Fig. 9 Biv; $n=6$ ), cyclopamine (Fig. 9Civ; $n=6$ )
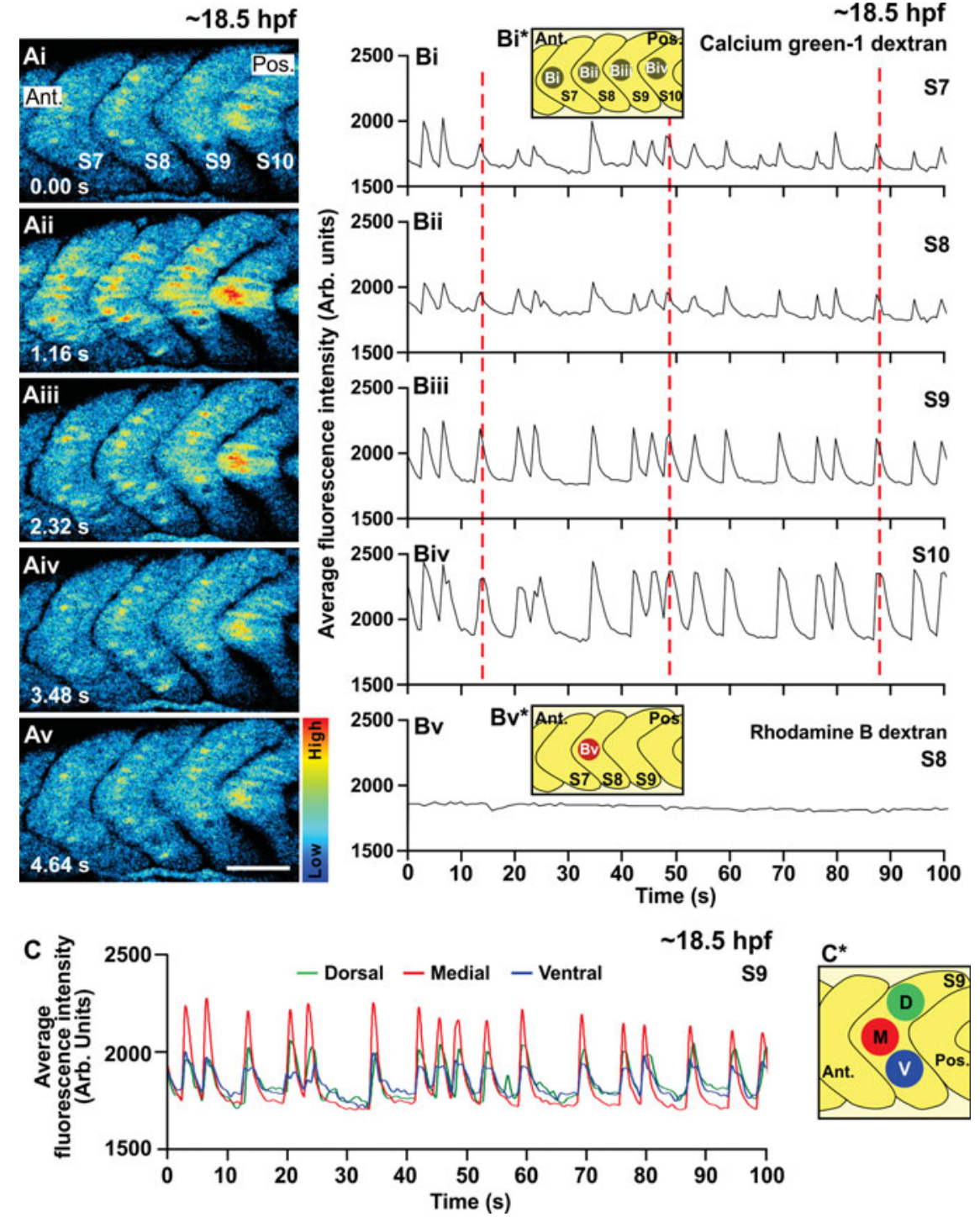


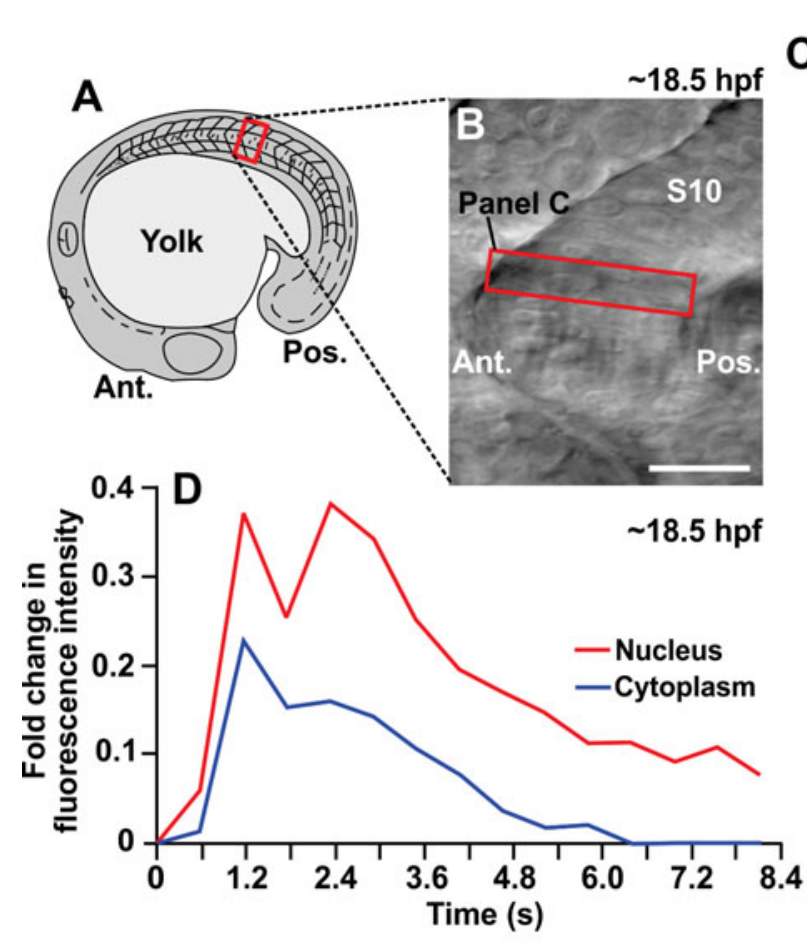

Fig. 7 (above). A Ca ${ }^{2+}$ signal generated in a single muscle cell in the trunk of an intact embryo at $\sim 18.5$ hpf (i.e., the 19-somite stage). Embryos $(n=3)$ were loaded with calcium green-1 dextran (10S) at the 1-cell stage and images were acquired via confocal microscopy. (A) Schematic diagram to show the position of somite 10 (red box) in the zebrafish embryo where the $\mathrm{Ca}^{2+}$ signal was acquired. (B) Bright-field image of somite 10 with the position of the imaged cell indicated (see red box). (C) Single confocal sections to show the $\mathrm{Ca}^{2+}$ signal generated in the imaged cell over a period of $\sim 8 \mathrm{~s}$. The images were acquired every $\sim 0.58 \mathrm{sec}$. The nucleus is outlined with a white dashed line in the first panel. The color scale represents the level of $\left[\mathrm{Ca}^{2+}\right]_{i}$, where red indicates a high level and blue indicates a low level. Ant. and Pos. are anterior and posterior, respectively. Scale bars are (B) $25 \mu \mathrm{m}$ and (C) $10 \mu \mathrm{m}$. (D) Temporal profile of the fold change in fluorescence intensity in the nucleus and cytoplasm in this muscle cell for the duration of the $\mathrm{Ca}^{2+}$ signal. $\sim 18.5$ hpf or forskolin (Fig. 9Div; $n=6$ ). These profiles show that the SP1 $\mathrm{Ca}^{2+}$ signals that normally occurred between $\sim 17.5$ to $19.5 \mathrm{hpf}$ in the untreated and DMSO-treated controls (Fig. 9 Aiv, Biv), were completely blocked in the cyclopamine- and forskolin-treated embryos (Fig. 9 Civ, Div). These results strongly suggest that the SP1 $\mathrm{Ca}^{2+}$ signals are generated exclusively by SMCs.

Slow muscle development and $\mathrm{Ca}^{2+}$ signaling in the trunk of $\mathrm{smo}^{-/-}$mutant embryos

As well as treating embryos with drugs to inhibit hedgehog signaling and thus the formation of the SMCs, a genetic approach, involving the homozygous $\mathrm{smo}^{-1}$ mutant (Barresi et al., 2000) was also used to confirm whether the SP1 $\mathrm{Ca}^{2+}$ signals were generated exclusively from SMCs (Fig. 10). The slow-muscle-omitted (smo) gene encodes the zebrafish smoothened protein, which (as mentioned previously) is a component of the hedgehog signaling pathway and is thus required for the development of the SMCs. In $\mathrm{smo}^{-/}$embryos, fast muscles develop normally; however, almost $99 \%$ of the SMCs are lost and the muscle pioneers are completely absent (Barresi etal., 2000). We found that at $24 \mathrm{hpf}$, the homozygous smo- embryos exhibited a curved trunk and tail, compared to normal control embryos (i.e., either wild-type or heterozygous $\mathrm{smo}^{+-} \mathrm{mu}$ tants; compare Fig. 10Bi with Fig. 10Ai). In addition, while the somites in the normal control embryos displayed the normal chevronshape (Fig. 10Aii), those in the $s m o^{-/}$em-
Fig. 8 (right). An example of the $\mathrm{Ca}^{2+}$ signals generated in the trunk of a wildtype embryo at $\sim 24$ hpf (i.e., the Prim-5 stage) as visualized by confocal microscopy using calcium green-1 dextran. (AiAiii) Representative $(n=3)$ single confocal sections to show the $\mathrm{Ca}^{2+}$ signals generated in the ventral half of somite 8 (see the schematic, panel $\left.A i^{*}\right)$. The time interval between each image was $0.68 \mathrm{sec}$. The color scale represents the level of $\left[\mathrm{Ca}^{2+}\right]_{i}$, where red indicates a high level and blue indicates a low level. Ant. and Pos. are anterior and posterior, respectively. Scale bar, 50 mm. (Bi) Temporal profile of the average calcium green-1 dextran fluorescence intensity (in Arb. units) recorded in an ROI covering 4-5 SMCs (i.e., $\sim 800 \mu^{2}$ ) placed in the ventral part of somite 8 of this wild-type embryo at $\sim 24$ hpf (see schematic, panel $B i^{*}$ ). (Bii) Temporal profile of average rhodamine $B$ dextran fluorescence intensity (in Arb. units) recorded in the same $\mathrm{ROI}$ over the same time period.

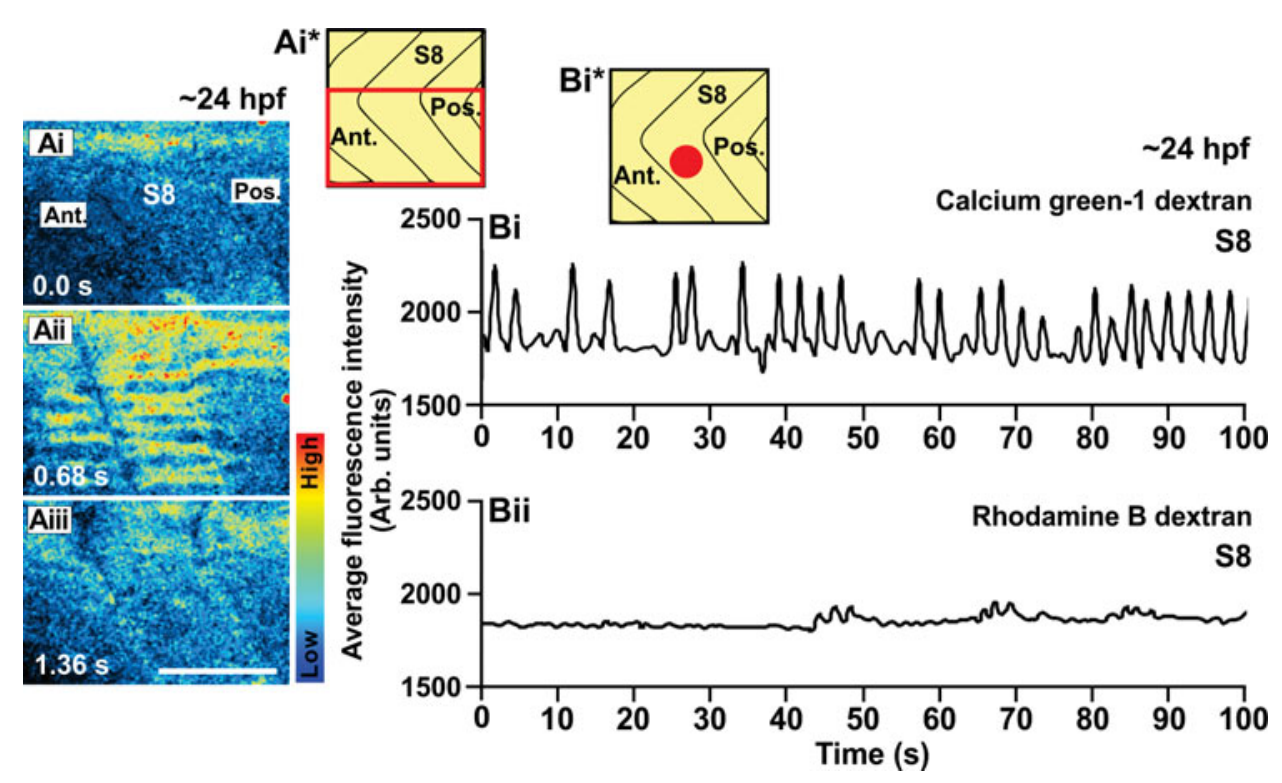


bryos were U-shaped (Fig. 10Bii). Immunohistochemistry of the slow muscle fibers using the F59 antibody showed that at $24 \mathrm{hpf}$ there was negligible slow muscle formation in the $\mathrm{smo}^{-/}$embryos (compare Fig. 10Biii with the normal control, Fig. 10Aiii). Fig. 10 Aiv and 10Biv show the profiles of aequorin-generated light from aeq-mRNA-injected normal control and $s m o^{-1}$ embryos, respectively. In these experiments, we used aeq-mRNA injected homozygous $\mathrm{smo}^{-}$embryos. The profiles of aequorin-generated light show that the SP1 $\mathrm{Ca}^{2+}$ signals that occurred at $\sim 17.5-19.5$ hpf in normal control embryos (Fig. 10Aiv; $n=5$ ) were completely absent in the $s m o^{-/}$embryos (Fig. 10Biv; $n=3$ ). These results support our cyclopamine and forskolin treatment experiments (Fig. 9), which together indicate that the SP1 $\mathrm{Ca}^{2+}$ signals are generated exclusively by SMCs.

\section{Effect of $\alpha$-bungarotoxin and nifedipine on the SP1 $\mathrm{Ca}^{2+}$ signals}

It has been previously reported that in zebrafish embryos, the trunk muscles start to show spontaneous contractile activity at $\sim 17 \mathrm{hpf}$ and these movements are generated by acetylcholine (ACh) release from motor nerve terminals (Grunwald et al., 1988;
Melançon et al., 1997). When acetylcholine binds to nicotinic acetylcholine receptors ( $\mathrm{nAChRs}$ ) expressed by the muscle cells, this binding is reported to activate dihydropyridine receptors (DHPRs) located on the surface membrane or on T-tubules, which in turn triggers the release of $\mathrm{Ca}^{2+}$ from intracellular stores via RyRs, and hence contraction occurs (Schneider and Chandler, 1973).

To investigate whether the nAChRs and DHPRs play a role in generating the SP1 $\mathrm{Ca}^{2+}$ signals, $\alpha$-actin-aeq transgenic embryos were incubated with either the nAChR blocker, $\alpha$-bungarotoxinAlexa Fluor 488 ( $n=3$; Fig. 11Bi) or the DHPR antagonist, nifedipine $(n=3$; Fig. $11 \mathrm{Ci})$ and the luminescence was compared with that generated by untreated controls (Fig. 11Ai). The distal $\sim 100 \mu \mathrm{m}$ portion of the tail of each embryo was excised with a tungsten needle just prior to incubation with the $\alpha$-bungarotoxin-Alexa Fluor 488 or nifedipine solution, in order to help facilitate the diffusion of drugs into trunk tissues containing the SMCs. The Alexa Fluor 488 moiety on $\alpha$-bungarotoxin was used to visualize and thus confirm the diffusion of the drug into the trunk via the tailcut (data not shown). We found that the SP1 $\mathrm{Ca}^{2+}$ signals were completely blocked by treatment with either $\alpha$-bungarotoxin or nifedipine right from the earliest time these signals appeared, which suggests that both $\mathrm{nAChRs}$ and DHPRs play a role in the initiation and generation of the SP1 $\mathrm{Ca}^{2+}$ signals in the trunk of zebrafish embryos. Moreover, the addition of triton X-100 at the end of each experiment (Fig. 11Aii-Cii) indicates that the inhibition of luminescence observed following the $\alpha$ bungarotoxin and nifedipine treatments was due to the drugs inhibiting the SP1 signals, rather than being due to a lack of active aequorin in the embryos.

Fig. 9. Effect of cyclopamine and forskolin on slow muscle development and $\mathrm{Ca}^{2+}$ signaling in the trunk. (Ai to Di) Representative bright-field images of embryos at 24 hpf that were either (Ai) untreated (controls), or else that were treated from 5.5 hpf with (Bi) 2\% DMSO (DMSO-control), (Ci) 0.1 $\mathrm{mM}$ cyclopamine or (Di) $0.15 \mathrm{mM}$ forskolin ( $n=4$ for each of the untreated and drug-treated embryos). (Aii-Dii) The somites of the embryos shown in (AiDi), respectively, are shown at higher magnification. (Aiii-Diii) Projected stacks of confocal images through the trunk (at the position of somite 8; S8) of embryos treated as described for (Ai-Di), respectively, prior to fixation at $24 \mathrm{hpf}$ and then the SMCS labeled by immunohistochemistry with the F59 myosin heavy chain antibody. Arrowheads in (Diii) indicate the few SMCs that still formed in the forskolin-treated embryos. Ant. and Pos. are anterior and posterior, respectively. Scale bars are (Ai-Di) 250 $\mu \mathrm{m}$, (Aii-Dii) $50 \mu \mathrm{m}$ and (Aiii-Diii) $25 \mu \mathrm{m}$. (Aiv-Div) Profiles of aequorin-generated light from $\alpha$-actin-aeq transgenic embryos treated as described for (Ai-Di), respectively, from $17 \mathrm{hpf}$ to $21 \mathrm{hpf}$ (i.e., the 16- to 24-somite stage). Data were plotted every 10 s, each data point representing $10 \mathrm{sec}$ of accumulated luminescence for an ROI covering the entire embryo (i.e., $1.24 \mathrm{~mm}^{2}$ ). 
The effect of 2-APB and ryanodine on the trunk $\mathrm{Ca}^{2+}$ signals

To investigate the mechanism of generation of the SP1 trunk $\mathrm{Ca}^{2+}$ signals, embryos were treated with the $\mathrm{IP}_{3} \mathrm{R}$ antagonist, 2APB, or the RyR antagonist, ryanodine (Fig. 12). The $\alpha$-actinaeq transgenic embryos were imaged using our PIMS starting at $\sim 16-17 \mathrm{hpf}$ and then they were treated with either $25 \mu \mathrm{M} 2$ APB or $50 \mu \mathrm{M}$ ryanodine for $3 \mathrm{hr}$ following the first appearance of the SP1 $\mathrm{Ca}^{2+}$ signals. Once again, $\sim 100 \mu \mathrm{m}$ of the distal portion of the tail of each embryo was excised just prior to incubation with the 2-APB, or ryanodine solution, in order to ensure that the drugs diffused into trunk tissues containing the SMCs. Fig. 12Ai shows a profile of aequorin-generated light in a representative $(n=9)$ untreated control embryo from $\sim 17 \mathrm{hpf}$ to $24 \mathrm{hpf}$ with the SP1 $\mathrm{Ca}^{2+}$ signals, the QP, and the SP2 $\mathrm{Ca}^{2+}$ signals indicated. A bright-field image of this embryo at $\sim 20.5$ hpf is shown in Fig. 12Aii. Fig. 12Bi is the profile of aequorin-

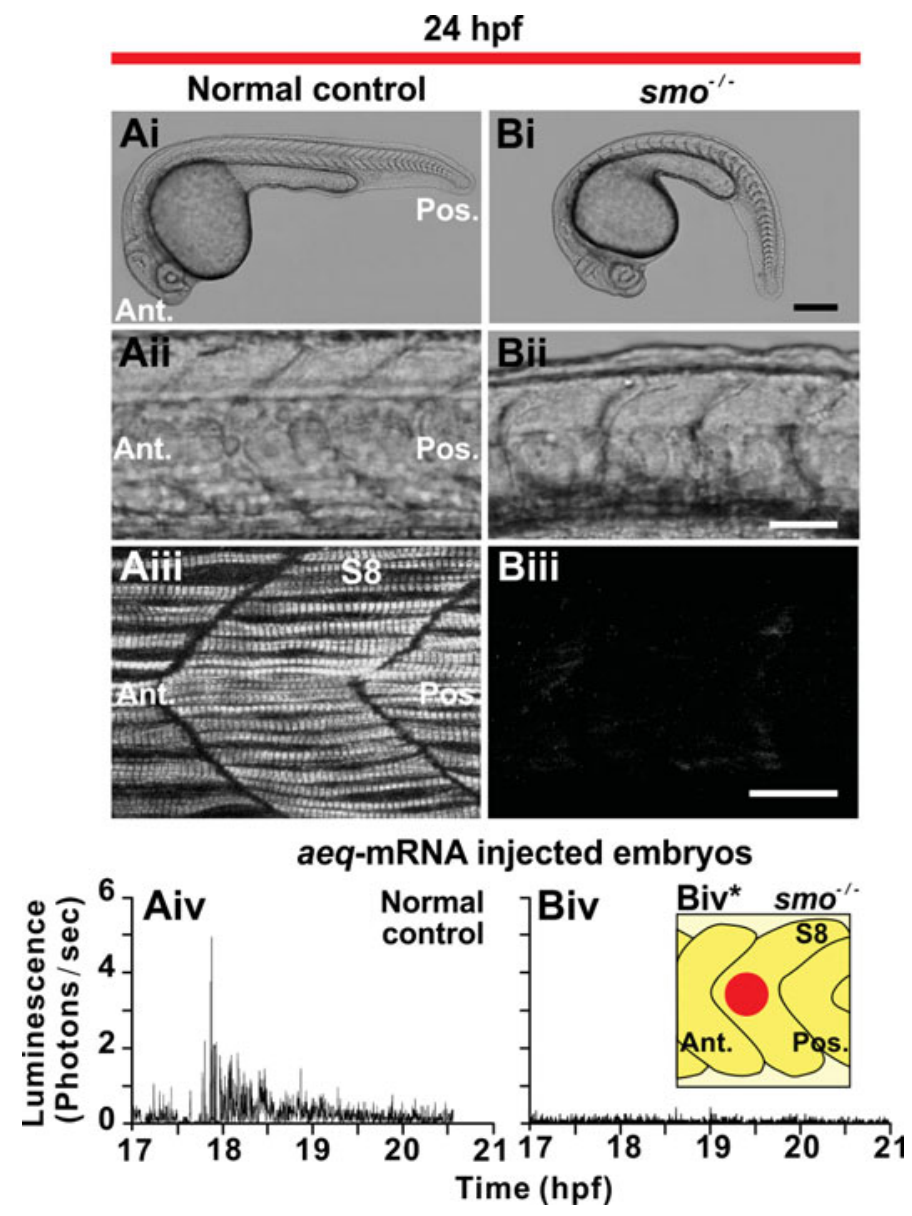

generated light in a representative embryo $(n=6)$ that was treated with 2-APB, which shows that the $\mathrm{SP} 1$ rapid $\mathrm{Ca}^{2+}$ spikes were blocked within $\sim 3 \pm 1.5$ min following the start of the 2 -APB treatment but that the SP2 $\mathrm{Ca}^{2+}$ spikes were still generated normally at $\sim 23 \mathrm{hpf}$. Treatment with 2-APB also inhibited the slow rising component of SP1. The gross morphology of the 2APB-treated embryo at $\sim 20.5 \mathrm{hpf}$ is shown in Fig. 12Bii, with the location of the tail-cut indicated by a black arrowhead. The aequorin burn-out experiment conducted at $\sim 24.5 \mathrm{hpf}$ (Fig. 12Biii) along with the appearance of the SP2 $\mathrm{Ca}^{2+}$ signals indicate that the low level of luminescence observed from $~ 17.5$ hpf to $23 \mathrm{hpf}$ following 2-APB treatment, was due to the drug inhibiting SP1 $\mathrm{Ca}^{2+}$ signals rather than being due to a lack of active aequorin in the trunk muscle cells.

Fig. $12 \mathrm{Ci}$ is a profile of aequorin-generated light in a representative embryo $(n=7)$ that was treated with ryanodine, which

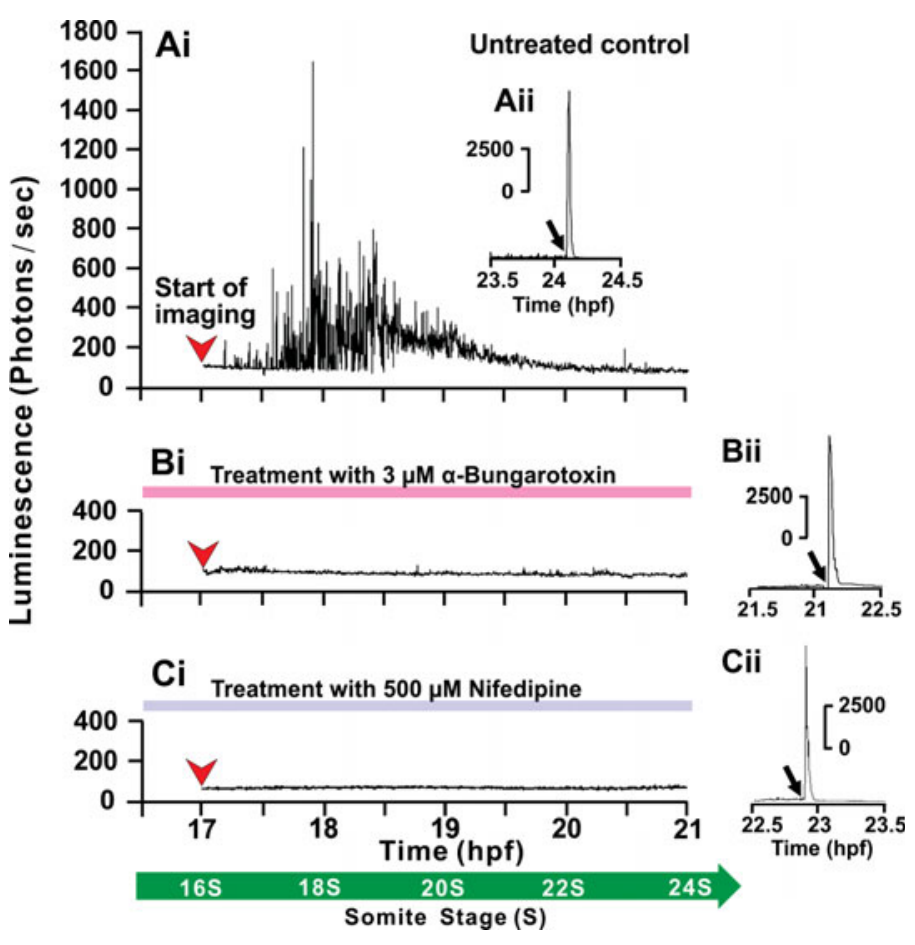

Fig. 10 (left). Slow muscle development and $\mathrm{Ca}^{2+}$ signaling in the trunk of smo $\%$ mutant embryos. (Ai,Bi) Representative bright-field images of (Ai) normal control $(n=3)$ or $(\mathbf{B i}) \mathrm{smo}^{-1-}$ mutant embryos $(n=3)$, at 24 hpf. (Aii,Bii) The somites of the embryos shown in (Ai, Bi), respectively, are shown at higher magnification. (Aiii,Biii) Projected stacks of confocal images through the trunk (at the level of somite 8; S8) of (Aiii) normal control and (Biii) $\mathrm{smo}^{-1-}$ mutant embryos that were fixed at $24 \mathrm{hpf}$ and then the SMCs labeled by immunohistochemistry with the F59 myosin heavy chain antibody. Ant. and Pos. are anterior and posterior, respectively. Scale bars are (Ai,Bi) $250 \mu \mathrm{m}$, (Aii,Bii) $50 \mu \mathrm{m}$ and (Aiii,Biii) $25 \mu \mathrm{m}$. (Aiv,Biv) Profiles of aequorin-generated light from aeq-mRNA injected (Aiv) normal (control) and (Biv) smo-- mutant embryos from $\sim 17 \mathrm{hpf}$ to $\sim 21 \mathrm{hpf}$ (i.e., the $\sim 16$ - to 24-somite stage). Data were plotted every $10 \mathrm{~s}$, with each data point representing $10 \mathrm{sec}$ of accumulated luminescence for an ROI covering $\sim 8000 \mu \mathrm{m}^{2}$ on somite 8 (Biv*).

Fig. 11 (right). Effect of $\boldsymbol{\alpha}$-bungarotoxin and nifedipine on the trunk $\mathbf{C a}^{2+}$ signals. Representative $(n=3)$ PMT-generated temporal profiles of the luminescence generated by $\alpha$-actin-aeq transgenic embryos that were either (Ai) untreated or else treated with (Bi) $3 \mu \mathrm{M} \alpha$-bungarotoxin or (Ci) 500 $\mu \mathrm{M}$ nifedipine from $16.5 \mathrm{hpf}$ to $21 \mathrm{hpf}$. All embryos had the tip of the tail removed prior to the treatment in order to enhance the diffusion of the drug into the trunk tissues. Data were collected every $10 \mathrm{sec}$ from $17 \mathrm{hpf}$ to $21 \mathrm{hpf}$. (Aii - Cii) Temporal profiles of the luminescence generated at the end of each imaging experiment when triton X-100 was added (see arrows) to the embryos in order to permeabilize the plasma membrane and thus expose any unspent cytoplasmic aequorin to extracellular $\mathrm{Ca}^{2+}$. 

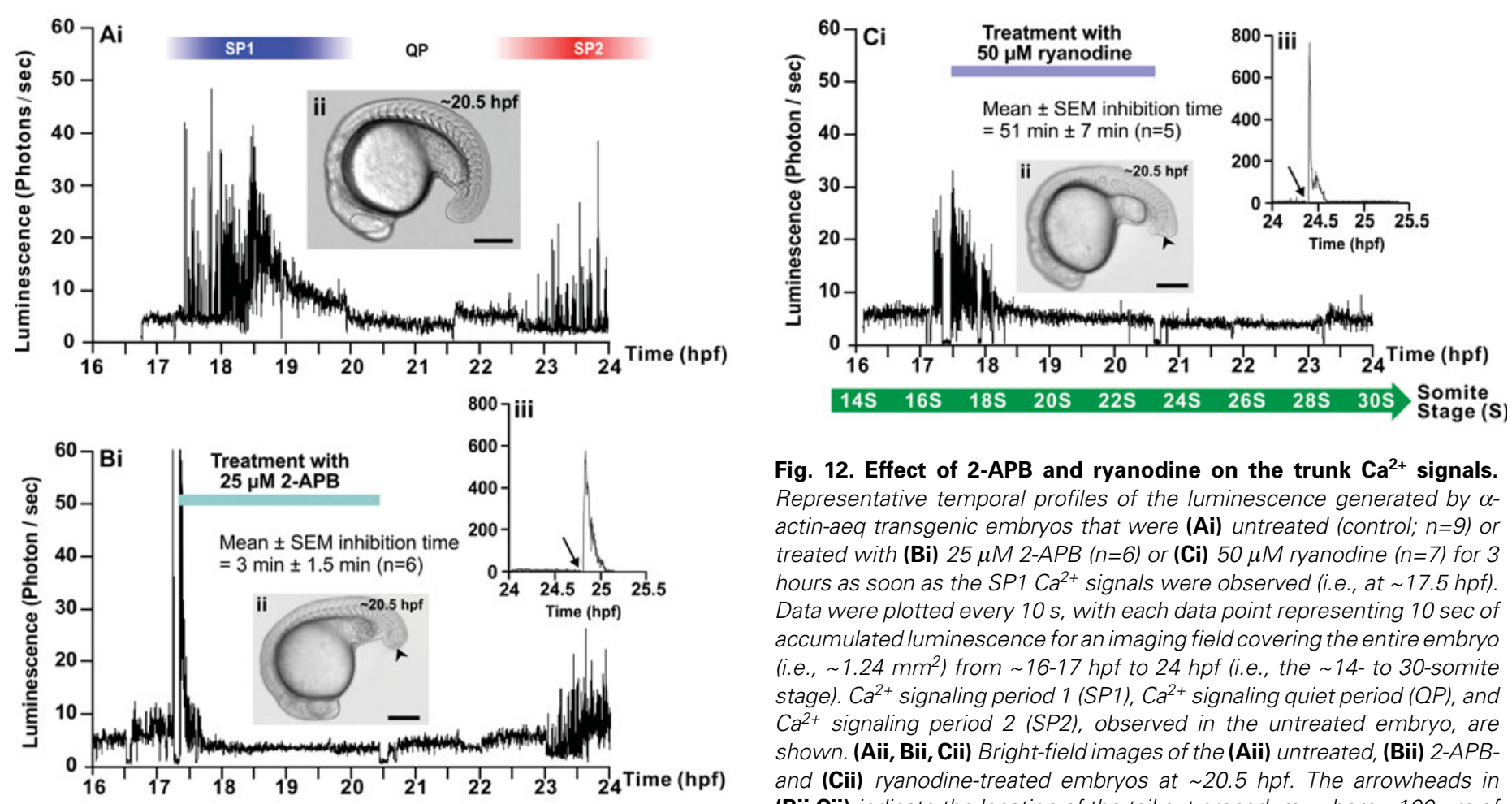

Fig. 12. Effect of 2-APB and ryanodine on the trunk $\mathrm{Ca}^{2+}$ signals. Representative temporal profiles of the luminescence generated by $\alpha$ actin-aeq transgenic embryos that were (Ai) untreated (control; $n=9$ ) or treated with (Bi) $25 \mu \mathrm{M} 2-A P B(n=6)$ or (Ci) $50 \mu \mathrm{M}$ ryanodine $(n=7)$ for 3 hours as soon as the SP1 $\mathrm{Ca}^{2+}$ signals were observed (i.e., at $\sim 17.5 \mathrm{hpf}$ ). Data were plotted every $10 \mathrm{~s}$, with each data point representing $10 \mathrm{sec}$ of accumulated luminescence for an imaging field covering the entire embryo (i.e., $\sim 1.24 \mathrm{~mm}^{2}$ ) from $\sim 16-17 \mathrm{hpf}$ to $24 \mathrm{hpf}$ (i.e., the $\sim 14$ - to 30-somite stage). $\mathrm{Ca}^{2+}$ signaling period $1(\mathrm{SP} 1), \mathrm{Ca}^{2+}$ signaling quiet period (QP), and $\mathrm{Ca}^{2+}$ signaling period 2 (SP2), observed in the untreated embryo, are shown. (Aii, Bii, Cii) Bright-field images of the (Aii) untreated, (Bii) 2-APBand (Cii) ryanodine-treated embryos at $\sim 20.5 \mathrm{hpf}$. The arrowheads in (Bii,Cii) indicate the location of the tail-cut procedure, where $\sim 100 \mu \mathrm{m}$ of the terminal portion of the tail bud was removed. This procedure was performed just prior to incubation with the drugs. Scale bars are $250 \mu$ m. (Biii,Ciii) Temporal profiles of the luminescence generated at the end of each imaging experiment when triton X-100 was added (see arrows) to the embryos in order to permeabilize the plasma membrane and thus expose any unspent cytoplasmic aequorin to extracellular $\mathrm{Ca}^{2+}$.

shows that the SP1 $\mathrm{Ca}^{2+}$ spikes were not blocked immediately following the start of treatment (i.e., just prior to $\sim 17.5 \mathrm{hpf}$ ) but that inhibition only occurred at $\sim 51 \pm 7$ min following the start of ryanodine treatment. Treatment with ryanodine also inhibited the slow rising component of SP1 that began at $\sim 18 \mathrm{hpf}$ in untreated controls. In addition, even though ryanodine treatment ended just before $\sim 21 \mathrm{hpf}$, the SP2 $\mathrm{Ca}^{2+}$ spikes that were normally generated at $\sim 23 \mathrm{hpf}$ were also significantly reduced. The gross morphology of the ryanodine-treated embryo at $\sim 20.5$ hpf is shown in Fig. 12Cii, with the location of the tail-cut once again indicated by a black arrowhead. An aequorin burnout experiment conducted just before 24.5 hpf (Fig. 12Ciii) indicates that the low level of luminescence observed following ryanodine treatment was due to the drug inhibiting the later portion of the SP1 signals and significantly reducing the SP2 $\mathrm{Ca}^{2+}$ signals, rather than being due to a lack of active aequorin in the embryo. When embryos were treated with ryanodine before the appearance of the SP1 $\mathrm{Ca}^{2+}$ signals (i.e., from $\sim 16.5$ hpf onward), once again the SP1 $\mathrm{Ca}^{2+}$ signals were only blocked after $\sim 18 \mathrm{hpf}$ (data not shown).

\section{Localization of $I P_{3} R s$ and RyRs in the developing trunk musculature}

Wild-type embryos were fixed at $16 \mathrm{hpf}, 18 \mathrm{hpf}, 20 \mathrm{hpf}, 22 \mathrm{hpf}$ and $24 \mathrm{hpf}$ ( $\mathrm{n}=3$ per time point) and then the $\mathrm{IP}_{3} \mathrm{Rs}$ were labeled via immunohistochemistry with the $I P_{3} R$ type 1 antibody (Fig. 13A). At $\sim 16 \mathrm{hpf}, \mathrm{IP}_{3} \mathrm{Rs}$ were expressed homogeneously throughout the SMCs (Fig. 13Ai). At $18 \mathrm{hpf}$ (i.e., during SP1), the $\mathrm{IP}_{3} \mathrm{Rs}$ began to organize into some clusters in the cytosol of
SMCs (see white arrowheads in Fig. 13Aii). At 20 hpf some $I_{3} R$ banding was observed in the cytosolic regions of the SMCs, and by $24 \mathrm{hpf}$, very distinct $\mathrm{IP}_{3} \mathrm{R}$ banding was observed throughout the entire length of the SMCs (Fig. 13 Aiii-Av). Fig. 13C shows representative images of SMCs at $24 \mathrm{hpf}$ that were dual labeled for both $\mathrm{IP}_{3} \mathrm{Rs}$ (Fig. 13Ci) and myosin (labeled with the F59 antibody; Fig. 13Cii). When these images were merged (Fig. 13Ciii), the $I P_{3} R$ s appeared to be expressed in the SR located between the myosin thick filament bands and thus were mainly associated with the sarcomeric I-bands. This labeling pattern is schematically represented in Fig. 13E.

In addition, embryos were fixed at $16 \mathrm{hpf}, 18 \mathrm{hpf}, 20 \mathrm{hpf}, 22$ hpf and $24 \mathrm{hpf}$ ( $\mathrm{n}=6$ per time point) and the RyRs were labeled via immunohistochemistry with the $34 \mathrm{C}$ antibody (Fig. 13B). No RyR expression was detected in the SMCs at $16 \mathrm{hpf}$ (i.e., prior to the start of SP1; Fig. 13Bi). At $18 \mathrm{hpf}$ (i.e., at the beginning of the slow rising phase of SP1), SMCs began to show a low level of homogeneous labeling (Fig. 13Bii). By $20 \mathrm{hpf}$, however, some RyR banding in the cytoplasmic region of the SMCs became apparent (see white arrowheads in Fig. 13Biii). At 22 hpf and $24 \mathrm{hpf}$ (i.e., during the QP and SP2, respectively), very distinct banding of RyRs was observed along the entire length of the SMCs (Fig. 13 Biv, Bv). When embryos at $24 \mathrm{hpf}$ were dual-labeled with $34 \mathrm{C}$ and rhodamine-phalloidin, to label the RyRs (Fig. 13Di) and F-actin (Fig. 13Dii), respectively, the superimposed RyR/F-actin images (Fig. 13Diii) showed that the RyR bands were approximately in the middle of the F-actin bands (see asterisks in Fig. $13 \mathrm{Di}$, Diii). This suggests that the RyRs were located in the SR located near to the Z-line. This is 

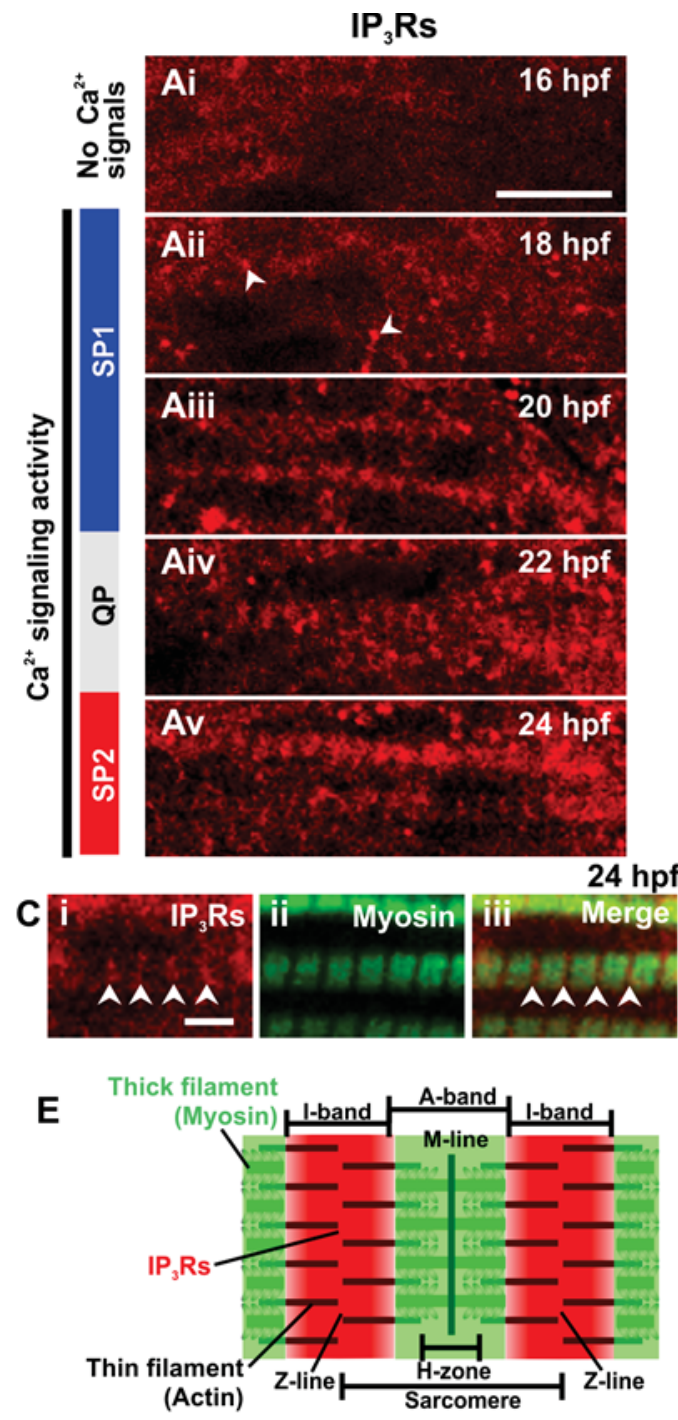
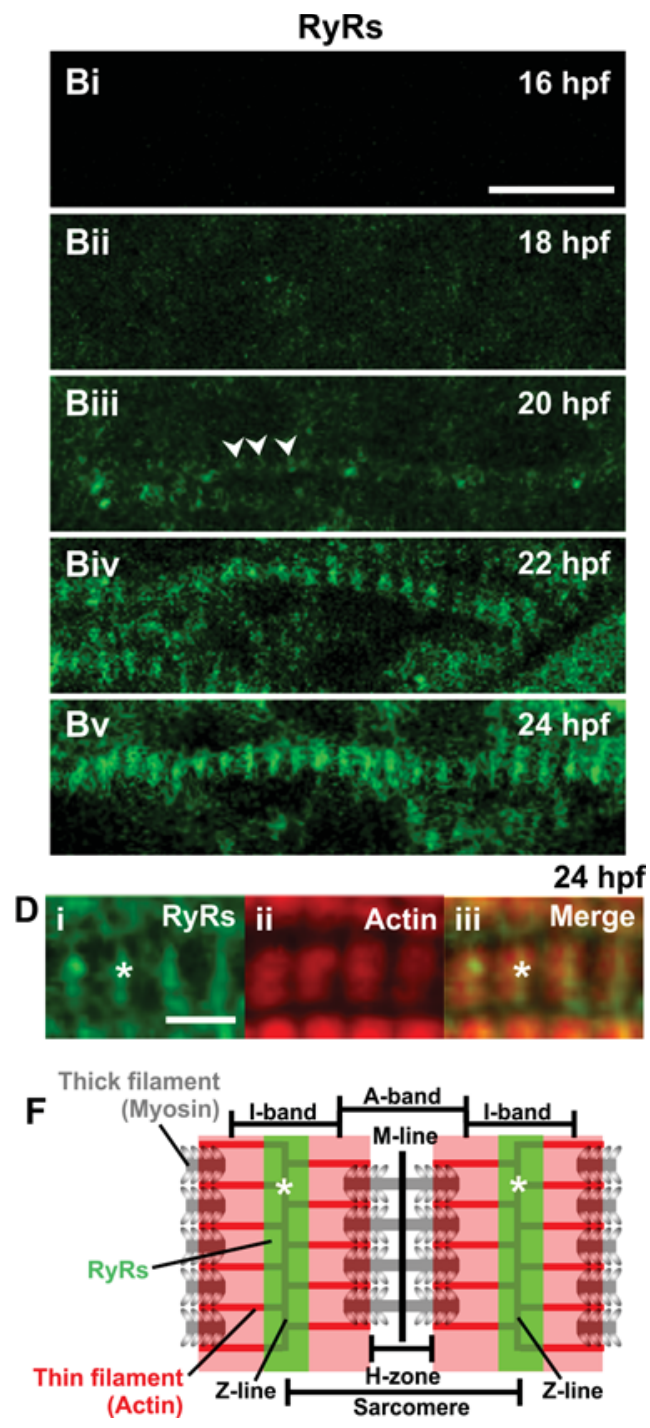

Fig. 13. Expression of $I P_{3} R$ s and RyRs in the trunk musculature. $(\mathbf{A}, \mathbf{B}) E m$ bryos were fixed at the times indicated in the upper right corner of each panel and then the (A) $I P_{3} R s$ and (B) RyRs were labeled via immunohistochemistry with the anti-Ins3R (Type 1) and 34C antibodies, respectively. Small clusters of $I P_{3} R s$ at 18 hpf (Aii), and RyRs at 20 hpf (Biii) are indicated with white arrowheads. (C) Dual labeling of (Ci) $I P_{3} R s$ and (Cii) myosin at 24 hpf. (Ciii) The $I P_{3} R$ and myosin images when merged. Arrowheads indicate the position of the $I P_{3} R s$ in the z-line SR. (D) Dual labeling of (Di) RyRs and (Dii) actin at 24 hpf. (Diii) The RyR and actin images when merged. Asterisks indicate the position of the RyRs. In (C,D), myosin was labeled via immunohistochemistry with the F59 antibody and actin was labeled with rhodamine phalloidin. (E,F) Schematic representations of a sarcomere to show (E) the pattern $I P_{3} R$ labeling with respect to myosin, and (F) the pattern of RyR labeling with respect to actin. Scale bars are $(A, B) 10$ $\mu \mathrm{m}$ and $(C, D) 3 \mu \mathrm{m}$.

14A). The low expression of myosin was relatively homogeneous within individual SMCs, with no labeling being observed in the regions of the vertical myoseptae (Fig. $14 \mathrm{Ai}$, Aiii). On the other hand, the level of labeling of $F$ actin was higher in the vertical myoseptae and in the end of the SMCs attached to these boundaries than in the more central re- schematically represented in Fig. 13F

Together, the results from Figs. 12 and 13 suggest that both $\mathrm{IP}_{3} \mathrm{Rs}$ and RyRs contribute to the SP1 $\mathrm{Ca}^{2+}$ signals, with $\mathrm{Ca}^{2+}$ release via $\mathrm{IP}_{3} \mathrm{Rs}$ being more dominant at the start of this signaling period (i.e., from $\sim 17.5 \mathrm{hpf}$ to $\sim 18 \mathrm{hpf}$ ), and that this is associated with the initial phase of low frequency SP1 $\mathrm{Ca}^{2+}$ signaling. $\mathrm{Ca}^{2+}$ release via RyRs becomes more prominent later (i.e., after $18 \mathrm{hpf}$ ) and thus perhaps contributes to both the higher frequency SP1 $\mathrm{Ca}^{2+}$ signaling as well as the slow rising $\mathrm{Ca}^{2+}$ component of SP1. In addition, the change in the pattern of $I P_{3} R s$ and RyRs is also reflected in the change in pattern of the $\mathrm{Ca}^{2+}$ signals generated by the SMCs. This transition is clearly indicated in Figs. 5, 6 and 8.

\section{The organization of myosin and F-actin in developing SMCs}

Wild-type embryos were fixed at $16 \mathrm{hpf}, 17 \mathrm{hpf}, 18 \mathrm{hpf}, 20$ hpf, $22 \mathrm{hpf}$ and $24 \mathrm{hpf}$ ( $\mathrm{n}=3$ per time point), and then myosin and F-actin were labeled with the F59 antibody and rhodaminephalloidin, respectively (Fig. 14). At $16 \mathrm{hpf}$, a relatively low level of myosin and actin labeling was observed in the SMCs (Fig. gions of the SMCs (Fig. 14 Aii, Aiii). At $17 \mathrm{hpf}$, the level of expression of both myosin and F-actin in the SMCs had increased significantly (i.e., compare Fig. 14 Bi, Bii, with Fig. 14 $\mathrm{Ai}, \mathrm{Aii})$ and both contractile proteins were beginning to be expressed along the whole length of a few individual SMCs (Fig. $14 \mathrm{Bi}-\mathrm{Biii}$ ) with the first hints of $\mathrm{F}$-actin banding being observed (see white arrowhead in Fig. 14Bii). At $18 \mathrm{hpf}$, a more prominent level of $\mathrm{F}$-actin banding was observed and the first clear signs of myosin banding appeared in the SMCs (Fig. 14Cii and $14 \mathrm{Ci}$, respectively). Furthermore, nearly all the SMCs showed expression of the two proteins. The appearance of these organized contractile proteins coincides with both the beginning of the slow rising phase of SP1, and the high frequency transients superimposed on this phase. From $\sim 20 \mathrm{hpf}$ to $24 \mathrm{hpf}$, the banding patterns of both $\mathrm{F}$-actin and myosin were clearly well formed along the entire length of each slow muscle cell (Fig. 14 Di-Diii, 14 Ei-Eiii and 14 Fi-Fiii). The organization of myosin and F-actin in a slow muscle cell at $24 \mathrm{hpf}$ is shown at higher magnification in Fig. 14 Gi-Giii and at higher magnification again in Fig. 14Giii* to show specific components of the sarcomere (labeled in the schematic in Fig. 14H). 
The effect of 2-APB and ryanodine on the organization of the trunk musculature and the formation of sarcomeres

Embryos were treated with various concentrations of 2-APB or ryanodine from $\sim 17 \mathrm{hpf}$ to $20 \mathrm{hpf}$ (i.e., from the 16- to 22somite stage), after which they were fixed and then the myosin and F-actin were labeled with the F59 antibody and rhodaminephalloidin, respectively ( $n=3$ for each treatment; Fig. 15). In the untreated controls (Fig. 15A) and the DMSO solvent controls (Fig. 15B), the trunk musculature and sarcomeres within the SMCs formed normally.

When embryos were treated with 2-APB at $25 \mu \mathrm{M}$ and $50 \mu \mathrm{M}$ (Fig. 15C and 15D, respectively), the gross morphology of the

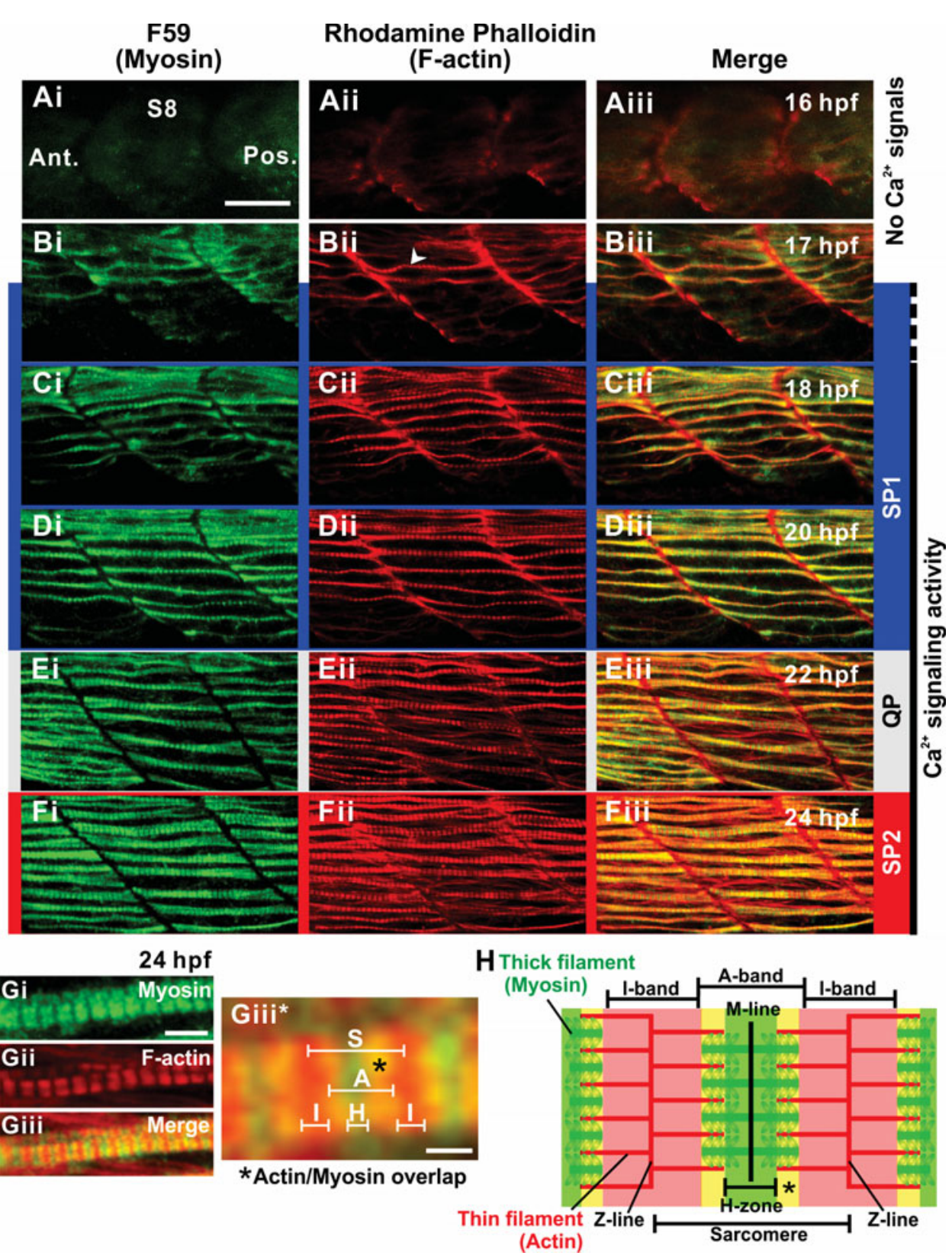

trunk musculature did not appear to be very different from that in the control embryos. Furthermore, the morphology of the SMCs themselves was also similar to the untreated and DMSOtreated controls. For example, they remained attached to the vertical myoseptae and spanned the entire myotome. At a sarcomeric level, however, the organization of the myosin thick filaments appeared to be disrupted to a greater extent than that of the actin thin filaments. For example, in Fig. 15Cii* and 15Dii* there is a clear degree of thin filament banding, whereas, in Fig. $15 \mathrm{Ci}^{*}$ and $15 \mathrm{Di}^{*}$ the banding of the myosin thick filaments appears to be disrupted in a concentration-dependent manner.

When embryos were treated with ryanodine at $10 \mu \mathrm{M}$ and 50 $\mu \mathrm{M}$, this caused significant abnormalities to both the myotome morphology and the organization of individual SMCs that increased in severity in a concentration-dependent manner (Fig. 15 $E, F)$. Treatment at both concentrations caused a severe disruption of the vertical myoseptae and as a result also to the SMCs spanning the gap between them. Embryos treated with $10 \mu \mathrm{M}$ ryanodine still possessed obvious SMCs but these were interspersed with bright aggregates of myosin and Factin that were largely located near the vertical myoseptae (Fig. 15 Ei-Eiii). This suggests that some of the outermost (with respect to the midline) SMCs had become detached at one end from the vertical myoseptae and had constricted or collapsed, forming brightlylabeled aggregates. At $50 \mu \mathrm{M}$, there were far fewer intact SMCs and far more bright aggregates (Fig. $15 \mathrm{Fi}$ Fiii). In addition, although at $10 \mu \mathrm{M}$ ryanodine, some sarcomeric banding was still observed in both the F-actin and myosin of the SMCs (Fig. $15 \mathrm{Ei}^{*}$ Eiii $^{*}$, at $50 \mu \mathrm{M}$, this banding was completely disrupted for both proteins (Fig. $15 \mathrm{Fi}^{\star}-\mathrm{Fiii}^{\star}$ ).

In order to examine the effect of 2 APB and ryanodine on the number of SMCs per myotome unit, these were quantified within somite 8 in both untreated and treated embryos. In the

Fig. 14. Expression of myosin and actin in the trunk musculature between $16 \mathrm{hpf}$ and 24 hpf. (A-F) Embryos were fixed at the times indicated in the upper right corner of each row of panels and then (Ai-Fi) the myosin was labeled via immunohistochemistry with the F59 myosin heavy chain antibody and (Aii-Fii) the F-actin was labeled with rhodamine phalloidin. (Aiii-Fiii) The merged myosin and F-actin images, with the overlapping regions shown in yellow. Ca ${ }^{2+}$ signaling periods 1 and 2 (SP1 and SP2), and the $\mathrm{Ca}^{2+}$ signaling quiet period (QP; from Fig. 3) are also shown. (Gi-Giii) Higher magnification views of (Fi-Fiii), respectively, showing the pattern of sarcomeric banding in the muscle fibers at 24 hpf. (Giii*) Higher magnification view of (Giii). S, A, H and I are sarcomere, A-band, H-zone and I-band, respectively. Scale bars are (A-F) $25 \mu \mathrm{m}$, (Gi-Giii) $5 \mu \mathrm{m}$ and (Giii*) $1 \mu \mathrm{m}$. (H) Schematic representation of a sarcomere. 
case of ryanodine-treated embryos, a brightly-labeled aggregate was considered to be a collapsed SMC and was thus counted as such. Fig. 16 shows that both $25 \mu \mathrm{m}$ and $50 \mu \mathrm{M}$ 2-APB resulted in a significantly lower number of SMCs in this somite at $p<0.001$, when compared with the controls, but that ryanodine (at both 10 $\mu \mathrm{M}$ and $50 \mu \mathrm{M}$ ) had no significant effect on SMC number.

\section{Discussion}

\section{Generation of muscle-specific apoaequorin-expressing trans- genic fish}

We previously developed protocols to express apoaequorin, the protein component of aequorin, transiently and ubiquitously in zebrafish embryos by microinjecting an apoaequorin-mRNA (aeq-mRNA) into 1-cell stage embryos. Active aequorin was then reconstituted in vivo by incubating the embryos from the 64-cell stage with the coelenterazine co-factor (Cheung et al., 2006). Although this transient aequorin expression approach successfully extends the aequorin-based $\mathrm{Ca}^{2+}$ imaging window by an additional $\sim 24$ hours (to $\sim 48 \mathrm{hpf}$ ), the aeq-mRNA is gradually degraded in the injected embryos resulting in a steady decline in the production of apoaequorin (Cheung et al., 2006). Furthermore, as the expression of aequorin is ubiquitous, and our current aequorin-based imaging platforms have no resolution in the z-axis, it is difficult to identify specific groups of cells, tissues or organ anlagen that are generating a particular signal in more complex, later stage embryos (Cheung et al., 2006). Thus, to follow-on from this transient, ubiquitous aequorin expression technique, we now report the successful generation of a transgenic zebrafish line that expresses apoaequorin targeted to the musculature using a muscle-specific $\alpha$-actin promoter.

Our new results show that this targeted apoaequorin is expressed as early as $\sim 12 \mathrm{hpf}$ (i.e., the 6-somite stage; Fig. 2B) and continues to be expressed until at least $48 \mathrm{hpf}$, allowing visualization of trunk-generated $\mathrm{Ca}^{2+}$ transients up to $\sim 52 \mathrm{hpf}$ (data not shown), which enables us to more precisely image $\mathrm{Ca}^{2+}$ signaling during trunk muscle development in a regular and reproducible manner. Consequently, we identified two distinct periods of spontaneous $\mathrm{Ca}^{2+}$ signaling generated exclusively by SMCs in the trunk musculature, which occur between 17.5 and $19.5 \mathrm{hpf}$ (SP1) and from 23 hpf onward (SP2), with $\mathrm{a} \mathrm{Ca}^{2+}$ signaling quiet period (QP) of around 3.5 hours duration between the two (Fig. 3Ai).

\section{Temporal characteristics of the SP1 and SP2 $\mathrm{Ca}^{2+}$ signals}

From the data acquired via aequorin-based $\mathrm{Ca}^{2+}$ imaging (using both transgenic embryos and aeq-mRNA injected embryos) we show that the SP1 and SP2 $\mathrm{Ca}^{2+}$ signals are generated within a restricted number of anterior somites and at specific times in a regular and reproducible manner. Although the occurrence of trunk $\mathrm{Ca}^{2+}$ signals between $\sim 17 \mathrm{hpf}$ and $\sim 24$ hpf have been reported previously using 


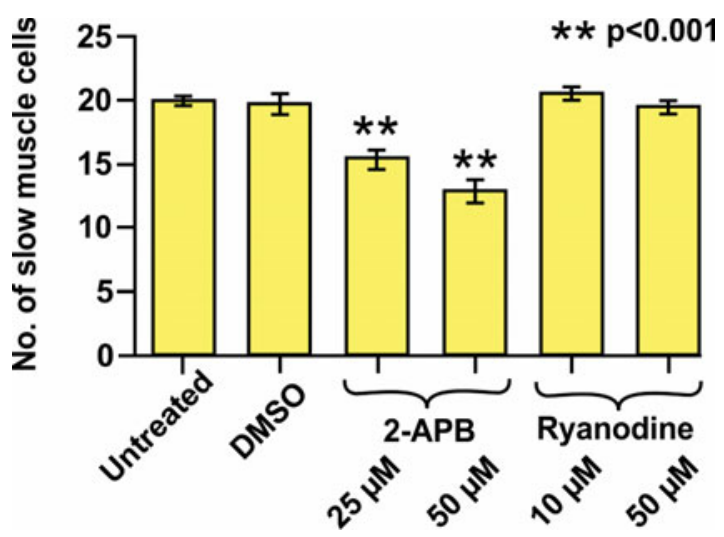

Fig. 16. Bar chart to show the effect of 2-APB and ryanodine on the number of SMCs in somite 8. Data are expressed as means \pm SEMs of 3-4 experiments. Asterisks indicate data that are significantly different from the DMSO control at $p<0.001$, as determined by the Student'st-test for unpaired samples.

aequorin as the $\mathrm{Ca}^{2+}$ reporter (Créton et al., 1998; Webb and Miller;2000; Cheung etal., 2006), the detailed characteristics and functions of these signals were not described or explored. Using fluorescent $\mathrm{Ca}^{2+}$ indicators, Brennan et al. (2005) subsequently reported and characterized (from a temporal perspective), a series of "nerve-mediated $\mathrm{Ca}^{2+}$ signals" that are generated in the SMCs of zebrafish from $\sim 17$ to $22 \mathrm{hpf}$. Our results confirm the generation of these signals, which correspond to what we have termed the SP1 $\mathrm{Ca}^{2+}$ signals, to distinguish them from the subsequent post-QP SP2 $\mathrm{Ca}^{2+}$ signals. The QP and the SP2 signals represent two new discoveries with respect to the dynamic $\mathrm{Ca}^{2+}$ signaling patterns generated during SMC development. Furthermore, the sudden appearance of the SP1 transients initially in somites 4 to 10 , follow a period of quiescent $\mathrm{Ca}^{2+}$ signaling as far as the zebrafish trunk is concerned. We have previously reported that a period of relatively localized stochastic $\mathrm{Ca}^{2+}$ signaling occurs in the formed somites and to a much lesser extent in the pre-somitic mesoderm during the early segmentation period, i.e., during the 1 to 8 somite stages ( 10 to $13 \mathrm{hpf}$; Leung et al., 2009). However, both we and others have reported that prior to the sudden appearance of the pan-somitic SP1 signals at $\sim 17.5 \mathrm{hpf}$, there is essentially a background resting level of $\mathrm{Ca}^{2+}$ within the zebrafish trunk (i.e., from all tissues) for at least 1 hour prior to the onset of SP1 (Figs. 3Ai, 12Bi and 12Ci; Gilland et al., 1999; Leung et al., 2009).

The data reported by Brennan et al. (2005) and our new observations correlate with the first of three different types of movement behavior reported by Saint-Amant and Drapeau (1998). The first of these movements consists of spontaneous, alternating left to right slow contractions of the trunk and tail, which were reported to begin suddenly at $\sim 17 \mathrm{hpf}$, with an initial average frequency of $\sim 0.57 \mathrm{~Hz}$. They peaked at $\sim 19 \mathrm{hpf}$ with a frequency of $\sim 0.96 \mathrm{~Hz}$, and then gradually decreased to $\sim 0.1 \mathrm{~Hz}$ per side of the embryo, i.e., one complete contraction cycle comprised of two alternating contractions, one on the left side, followed by one on the right side (Saint-Amant and Drapeau, 1998).

The maximum $\mathrm{Ca}^{2+}$ signaling frequency we recorded on one side of the embryo at $\sim 19 \mathrm{hpf}$, however, was $\sim 0.12 \mathrm{~Hz}$, i.e., almost an order of magnitude slower than the contraction frequency reported by Saint-Amant and Drapeau (1998). In both cases embryos were dechorionated as part of the experimental protocol to improve imaging. Saint-Amant and Drapeau (1998), however, immobilized just the head of the embryos with low melting point agarose, thus allowing the trunk and tail to move freely, whereas in our experiments, embryos were immersed in 3\% methyl cellulose for the duration of imaging to prevent them moving out of the field of view. This may have restricted the degree of trunk/tail contraction, and thus explain the lower rates of $\mathrm{Ca}^{2+}$ signals observed during our imaging experiments. However, the onsettimes of the trunk/tail contractions reported by Saint-Amant and Drapeau (1998), and the spontaneous $\mathrm{Ca}^{2+}$ signaling activities that we visualized (i.e., at $\sim 17$ and $\sim 17.5 \mathrm{hpf}$, respectively), are remarkably similar. Indeed, while our aequorin-based imaging system did not allow us to record whether or not a trunk/tail contraction accompanied a $\mathrm{Ca}^{2+}$ signal, we were able to confirm this correlation during our confocal fluorescent $\mathrm{Ca}^{2+}$ imaging experiments, (see Supplemental Movie \#1).

We also report an additional new observation regarding a slow rising component of $\mathrm{SMC} \mathrm{Ca}{ }^{2+}$ activity during SP1, that started at $\sim 18 \mathrm{hpf}$, reached a maximum at $\sim 18.75 \mathrm{hpf}$ and then returned to its original resting value by $\sim 19.5-20 \mathrm{hpf}$ (Fig. 3Ai). At present, we do not understand the significance or developmental function of the slow rising $\mathrm{Ca}^{2+}$ component. It could, however, be completely abolished via treatment with either 2-APB or ryanodine (Figs. $12 \mathrm{Bi}, \mathrm{Ci})$ suggesting a dual generation mechanism that involves $\mathrm{Ca}^{2+}$ release from both receptor types. The appearance of the slow rising component correlates with the beginning of the higher frequency oscillations of SP1 (Fig. 3Ai), the expression of RyRs (Fig. 13Bii) and the appearance of organized sarcomeres in all the SMCs (Fig. 14Ciii). A somewhat similar slow rising $\mathrm{Ca}^{2+}$ component, with accompanying super-imposed $\mathrm{Ca}^{2+}$ spiking, has been reported during the process of neural induction in Xenopus development (Leclerc et al., 2000). It was subsequently shown that these $\mathrm{Ca}^{2+}$ signals correlated with the expression pattern of the early neural precursor gene, Zic3 (Leclerc et al., 2003).

SP1 ends at $\sim 19.5 \mathrm{hpf}$ (Fig. 3), as embryos enter into the QP. We are assuming, therefore, that zero $\mathrm{Ca}^{2+}$ signaling activity in the slow muscle between 19.5 and 23 hpf equates to no contractile activity during this period. Saint-Amant and Drapeau (1998) do report that in their methyl cellulose-free set-up, the frequency of the spontaneous trunk/tail contractions begins to fall following a peak at $\sim 19 \mathrm{hpf}$. However, they also describe the onset, at $\sim 21$ hpf, of a second type of locomotor behaviour, which they called a "touch-evoked" response. Under our experimental conditions, embryos were never subjected to any touch stimulation. Furthermore, our methyl cellulose embedding protocol may have in some way speeded up the fall in spontaneous contractile activity, leading to the establishment of the QP at $19.5 \mathrm{hpf}$.

It is interesting to note, however, that the QP of $\mathrm{Ca}^{2+}$ signaling from the SMCs coincides with a period (i.e., from 21 to $22 \mathrm{hpf}$ ) of intense spontaneous $\mathrm{Ca}^{2+}$ signaling activity within the developing spinal cord of the zebrafish embryo (Ashworth and Bolsover, 2002). We failed to detect these spinal cord transients either due to the tissue-specificity of apoaequorin expression in our aequorinbased imaging experiments, or due to the focal plane selected for the fluorescence-based confocal scanning. Thus, the QP appears to correlate with a transition from spontaneous contractile 
activity that does not require sensory input and motor activity circuitry, to touch-response contractile activity that does. Perhaps it is not surprising, therefore, that developmental $\mathrm{Ca}^{2+}$ signaling activity in the embryonic spinal column increases during this period while the required circuitry and contacts are being established, while that in the SMCs themselves, declines.

A second period of spontaneous $\mathrm{Ca}^{2+}$ signaling, the SP2, begins at $\sim 23 \mathrm{hpf}$. The frequency, duration, and spatial signature of the SP2 signals are quite distinct from those of the early SP1 signals. Brennan et al. (2005) only reported signaling activity up to $22 \mathrm{hpf}$, thus our new study extends the visualization of trunk muscle $\mathrm{Ca}^{2+}$ signaling into the SP2 period. Saint-Amant and Drapeau (1998) report that in older embryos (i.e., >24 hpf) the spontaneous contractions change from being continuous to occurring in bursts of between 3-5 coils, followed by periods of inactivity. These might prove to correlate with the bursts of $\mathrm{Ca}^{2+}$ signaling activity followed by periods of inactivity that we observed to start at $23 \mathrm{hpf}$ (Fig. 3Ci).

The distinct spatiotemporal pattern of our SP1 and SP2 $\mathrm{Ca}^{2+}$ signals bare some similarities to the Xenopus primary myocytegenerated LDTs and SDTs, respectively, reported by Campbell et al. (2006). The durations of the SP1 signals and LDTs, i.e., 5-10 sec and $\sim 80$ secs, respectively, and SP2 signals and SDTs, i.e., $\sim 0.6 \mathrm{sec}$ and $\sim 2 \mathrm{sec}$, respectively are, however, significantly different. This may reflect species differences, but is more likely due to the fact that we were recording the transients in situ, rather than in cell culture, where a number of endogenous in situ feedback mechanisms that are absent in the simplified cell culture system, might restrict the duration of the transients. What is similar is the fact that both the SP1 signals and the LDTs are reported to have a nuclear component, and it was proposed that in both cases the signals might regulate gene expression and subsequent sarcomeric assembly events. Furthermore, Campbell et al. (2006) reported that in Xenopus myocytes, LDT production ceases, and only SDTs persist during a period of rapid sarcomeric assembly. We report a somewhat similar pattern of signaling in intact zebrafish, where the nuclear component of signaling is restricted to SP1, and after the QP, only short duration cytoplasmic signals persist during the SP2. Although Campbell et al. (2006) hypothesized that LDTs might also involve $\mathrm{Ca}^{2+}$ release from IP $R$ s, we show that this is indeed the case with regard to the early SP1 signals (Fig. 12). We did not, however, examine our SP2 signals at a high enough magnification (due to the depth of the SMCs within an intact zebrafish embryo) to determine whether these signals were restricted to the subplasmalemmal space as was the case with the SDTs reported from isolated Xenopus myotubules (Campbell et al., 2006).

Rostrocaudal spatial characteristics of the SP1 $\mathrm{Ca}^{2+}$ signals

Our data clearly demonstrate that at $\sim 17.5 \mathrm{hpf}$, the low frequency early SP1 signals were only generated from S4 to S10. At $\sim 18.5 \mathrm{hpf}$, the higher frequency SP1 signals were seen to extend rostrally into $S 2$ and S3 (but never S1) and caudally to S12 (Fig. $4 C, D)$. We have evidence from our addition of triton X-100 to aequorin-expressing embryos at various times during slow muscle cell differentiation, to show that luminescence is generated along the entire length of the trunk musculature and not just from S2S12 (data not shown). Our aequorin burn-out data suggest that even though the normal $\mathrm{Ca}^{2+}$ signals are only generated between
S2-S12, the stable expression and reconstitution of aequorin does extend to the other somites too. This was found to be the case for both the transgenic embryos $(n=6)$ and where the aequorin is transiently expressed $(n=3)$. In teleosts, the first three somites (i.e., S1-S3) are regarded as giving rise to mainly occipital and other head structures (Ma et al., 2009). Thus, the most rostral somite that gives rise to truly axial structures, including the trunk musculature, has been suggested to be S4 (Holley and NüssleinVolhard, 2000; Ma et al., 2009). We propose therefore that a possible reason why the initial low-frequency SP1 $\mathrm{Ca}^{2+}$ signals (i.e., at $\sim 17.5 \mathrm{hpf}$ ) are not seen in S1 to S3, might be because the precursor cells (including the SMCs) in these somites are destined to have different developmental fates than the more caudal somites. The higher frequency SP1 $\mathrm{Ca}^{2+}$ signals that start at $\sim 18.5$ hpf do extend into S2 and S3, which again may reflect differences in the type of SMCs derived from these somites. The caudal restriction of the SP1 signals (i.e., S10 and S12 at 17.5 hpf and $\sim 18.5 \mathrm{hpf}$, respectively) may reflect the fact that this block of more anterior somites differentiates and matures first. This also fits in well with observations made by Saint-Amant and Drapeau (1998) that only a restricted number of more anterior somites appear to be active in generating spontaneous contractile movements. Furthermore, the anterior trunk somites have been described as being genetically different from the tail somites, although the primary mechanism for somite formation appears to be the same for both anterior trunk and tail somites (Szeto and Kimelman, 2006; Holley, 2006). We suggest that these differences reflect, and/or maybe the result of differences in the embryonic $\mathrm{Ca}^{2+}$ signaling signature.

\section{Sub-cellular spatial characteristics of the SP1 and SP2 $\mathrm{Ca}^{2+}$ signals}

Our new imaging data further revealed that the SP1 signals also had distinct spatial characteristics with regard to where they were generated within a differentiating SMC. At $\sim 17.5 \mathrm{hpf}$, the low frequency $\mathrm{Ca}^{2+}$ signals appeared to be generated mainly in and around the nucleus with relatively little spread out into the cytoplasm (Fig. 5). At $18.5 \mathrm{hpf}$, however, it is clear that although nuclear signaling still plays a significant role, the $\mathrm{Ca}^{2+}$ signals also propagate out into the cytosolic fraction of the SMC (Figs. 6 and 7 ). It is also clear that all the muscle cells within a single somite, and the muscle cells in adjacent somites, "fire" their $\mathrm{Ca}^{2+}$ signals at approximately the same time (Fig. 6). This indicates synchronized activity and suggests a role for neuronal-based co-ordination of the $\mathrm{Ca}^{2+}$ signaling in these somites, thus supporting the observations of Brennan et al. (2005). The synchronized $\mathrm{Ca}^{2+}$ activity in S7 to $\mathrm{S} 10$ at $\sim 18 \mathrm{hpf}$ is tightly correlated with a spontaneous rostral/caudal contraction of these somites (see Supplemental Movie \#1).

Fig. 7 also clearly indicates that within an individual slow muscle cell at $\sim 18.5 \mathrm{hpf}$, the $\mathrm{Ca}^{2+}$ rises in the nuclear region first then it spreads rapidly to the extremities of the elongated slow muscle cell. In the example shown, between the image taken at $0.58 \mathrm{sec}$ and that at $1.16 \mathrm{sec}$, a duration of $0.58 \mathrm{sec}$, the $\mathrm{Ca}^{2+}$ signal has spread from the nucleus to the right hand extremity of the cell, a distance of $\sim 20 \mu \mathrm{m}$. At the image acquisition rate employed, this represents a minimum propagation velocity of $\sim 34$ $\mu \mathrm{m} / \mathrm{sec}$, which is similar to the velocity of cytoplasmic $\mathrm{Ca}^{2+}$ waves, i.e., $56 \pm 9 \mu \mathrm{m} / \mathrm{sec}$, reported from rat myotubes generated from a 
primary culture of skeletal muscle (Jaimovich et al., 2000). The $\mathrm{Ca}^{2+}$ signal then dies down in the cytoplasmic regions of the cell after $\sim 2$ secs, but lingers longer in the nucleus, i.e. in this particular example, for at least 7.5 secs (Fig. 7).

The complex spatial nature of the spontaneous $\mathrm{Ca}^{2+}$ signals shown in Fig. 7 also bear a strong similarity to aspects of $\mathrm{Ca}^{2+}$ signals induced by elevated $\mathrm{K}^{+}$in cultured rat and mouse myotubes (Jaimovich et al., 2000; Powell et al., 2001). In these cells two types of $\mathrm{Ca}^{2+}$ transient, with different kinetics were identified. One was a fast $\mathrm{Ca}^{2+}$ transient, which was associated with RyRs and excitation-contraction coupling (E-CC). Following this fast transient was a slower $\mathrm{Ca}^{2+}$ transient, which resulted in an increase in nucleoplasmic $\mathrm{Ca}^{2+}$ (that lasted in the range of 30-40 secs), but not in contraction. We suggest that something similar is observed in zebrafish SMCs (Fig. 7) where the shorter duration cytoplasmic signals are associated with E-CC and the longer duration nuclear signals serve another function. There would also appear to be clear differences between our new observations in intact zebrafish and those from cultured mouse myotubes. For example, we observe a rise in nuclear $\mathrm{Ca}^{2+}$ before the generation of the cytosolic transient (Fig. 7).

In addition, it has been reported that treatment of rat and mouse myotubes with nifedipine or 2-APB completely blocked the slow nucleoplasmic transient, thus suggesting that DHPRs are the main membrane voltage sensors for this $\mathrm{Ca}^{2+}$ signal, and that the signal was generated mainly by $\mathrm{Ca}^{2+}$ release from internal stores via $\mathrm{IP}_{3} \mathrm{Rs}$ (Powell et al., 2001; Jaimovich and Carrasco, 2002). Furthermore, this slow $\mathrm{IP}_{3} \mathrm{R}$-mediated $\mathrm{Ca}^{2+}$ transient was reported to be linked to developmental gene expression, where $\mathrm{K}^{+}$-induced depolarization of mouse myotubes resulted in the rapid (i.e., within $30 \mathrm{sec}-10 \mathrm{~min}$ ) phosphorylation of the mitogen activated kinases, ERK $1 / 2$ and the transcription factor, CREB, as well as the expression (within 5-15 min) of various early genes (Powell et al., 2001; Jaimovich and Carrasco, 2002; Carrasco et al., 2003; Cárdenas et al., 2005). Thus, we suggest that our early $\mathrm{Ca}^{2+}$ oscillations located in and around the nucleus of SMCs at $\sim 17.5 \mathrm{hpf}$ (Fig. 5A), as well as the longer duration nuclear component of the later signals observed at $\sim 18.5 \mathrm{hpf}$ (Figs. $6 \mathrm{~A}$, $7 \mathrm{C})$, may play a role in gene expression and the translation of some important proteins during myofibrillogenesis, whereas the rapid cytoplasmic phase of the SMC generated signals might play a role in the spontaneous E-CC along the anterior trunk (Supplemental Movie \#1).

In the case of the spontaneous SP2 $\mathrm{Ca}^{2+}$ signals, we did not visualize any specific long-duration nuclear component, as the SP2 signals consisted exclusively of short-duration cytoplasmic signals (Fig. 8). The SP2 $\mathrm{Ca}^{2+}$ signals were generated only when the banding patterns of $\mathrm{F}$-actin and myosin were clearly formed along the entire length of each SMC (Fig. 14 Fi-Fiii); when the $\mathrm{IP}_{3} \mathrm{Rs}$ and RyRs were well organized into distinct bands (Fig. $13 \mathrm{Av}, \mathrm{Bv})$; and they were well-correlated with stronger contractions within the SMCs of the trunk.

\section{Confirming the specific slow muscle cell location of the SP1 $\mathrm{Ca}^{2+}$ signals}

From observations using confocal $\mathrm{Ca}^{2+}$ imaging, the $\mathrm{SP} 1 \mathrm{Ca}^{2+}$ signals appeared to be generated exclusively in SMCs. This was tested and confirmed both pharmacologically and genetically. In the case of the former, embryos were treated with cyclopamine or forskolin (Fig. 9), while in the case of the latter, we imaged the $\mathrm{Ca}^{2+}$ signaling signature of $\mathrm{smo}^{-/}$mutant fish (Fig. 10). In all three cases, embryos displayed U-shape somites (Figs. 9Cii,Dii, 10Bii) and the SMCs did not develop (Figs. 9Ciii,Diii, 10Biii) as previously reported by (Barresi et al., 2000; 2001). As no $\mathrm{Ca}^{2+}$ signals were generated from the trunk of these embryos (Figs. 9Civ,Div, 10Biv) we concluded that the normal pattern of $\mathrm{Ca}^{2+}$ signals observed in the untreated wild-type embryos are generated exclusively by the SMCs.

We did not, via either aequorin- or fluorescence-based imaging, visualize any $\mathrm{Ca}^{2+}$ signals from the fast muscle cells (FMCs). This was in spite of the aequorin being clearly expressed in the FMC precursors (Fig. 2C) and confocal optical sectioning through the entire myotome block of calcium green-1 dextran-loaded embryos (data not shown). This result was somewhat puzzling as FMCs have been reported to differentiate immediately after the medial-to-lateral migration of the SMCs, which begins at $\sim 20 \mathrm{hpf}$ (i.e., the 21-somite stage) in the anterior somites (Blagden et al., 1997). Therefore, our $\mathrm{Ca}^{2+}$ signaling data seems to suggest that the spontaneous contractions of the embryonic trunk up to at least $24.5 \mathrm{hpf}$ (i.e., when imaging was terminated) are generated by SMCs alone.

\section{Exploring the mechanisms of $\mathrm{Ca}^{2+}$ release in SMCs}

Our new data showed that when embryos were treated with 25 $\mu \mathrm{M} 2$-APB as soon as the first SP1 $\mathrm{Ca}^{2+}$ signals were observed (i.e., at $\sim 17-17.5 \mathrm{hpf}$ ), then these signals were blocked within $\sim 3$ $\pm 1.5 \mathrm{~min}(\mathrm{n}=6)$ of treatment (Fig. 12Bi). On the other hand, when embryos were treated with $50 \mu \mathrm{M}$ ryanodine as soon as the first $\mathrm{SP} 1 \mathrm{Ca}^{2+}$ signals were observed, then initially only the amplitude of SP1 $\mathrm{Ca}^{2+}$ signaling was reduced. Complete inhibition of the SP1 signals only occurred $\sim 51 \mathrm{~min} \pm 7 \mathrm{~min}(\mathrm{n}=5)$ after treatment (Fig. 12Ci), i.e., some 17-fold slower than treatment with 2-APB. These data suggest, therefore, that the initial high amplitude, low frequency SP1 $\mathrm{Ca}^{2+}$ signals that were visualized mainly in and around the nucleus at $\sim 17.5 \mathrm{hpf}$, may be generated primarily via $\mathrm{IP}_{3} \mathrm{Rs}$ rather than RyRs. The latter then become more prominent in generating the higher frequency cytoplasmic signals that start after $\sim 18$ hpf. We do not propose, however, that either one of these two $\mathrm{Ca}^{2+}$ release mechanisms operates to the exclusion of the other during these time periods, but rather that one plays a more prominent role with regard to the method of $\mathrm{Ca}^{2+}$ release at a particular developmental time and in a specific cellular location. We suggest that the identification of functional $\mathrm{IP}_{3} \mathrm{Rs}$ generating spatially and temporally distinct sub-cellular $\mathrm{Ca}^{2+}$ transients during the early stages of SMC differentiation in intact zebrafish is one of the significant new findings presented in this paper.

In addition to treatment with different antagonists of $\mathrm{Ca}^{2+}$ release from intracellular stores, we report that the SP1 $\mathrm{Ca}^{2+}$ signals could be completely blocked from their very earliest appearance, i.e., from $\sim 17.5 \mathrm{hpf}$ onward, by treatment with either the acetylcholine receptor (AchR) antagonist, $\alpha$-bungarotoxin (Fig. 11Bi) or the DHPR antagonist, nifedipine (Fig. 11Ci). Brennan et al. (2005) also showed that somewhat later, i.e., between 18$20 \mathrm{hpf}$, the $\mathrm{Ca}^{2+}$ signals and spontaneous muscle contractions could be completely blocked with $\alpha$-bungarotoxin, and that $>21$ hpf the muscle contractions were significantly blocked with nifedipine. We felt it most important to establish that treatment with $\alpha$-bungarotoxin and nifedipine could block the very earliest of 
the SP1 transients, thus establishing a clear developmental timecourse with respect to the relationship between neuronal innervation of the somites and the generation of $\mathrm{Ca}^{2+}$ transients by the SMCs. In addition, Saint-Amant and Drapeau (1998) reported that lesioning the hindbrain had no effect on the generation of the spontaneous contractions and they suggested that these contractions resulted from activation of a primitive spinal circuit. Indeed, our $\alpha$-bungarotoxin and nifedipine data support this suggestion, and we propose that acetylcholine induces periodic depolarizations of the sarcolemma of SMCs, which, via the activation of DHPRs, stimulates the release of $\mathrm{Ca}^{2+}$ from intracellular stores initially by $\mathrm{IP}_{3} \mathrm{Rs}$, then mainly RyRs, and thus generates the periodic spontaneous trunk contractions in zebrafish (Supplemental Fig. S1).

\section{Localization of IP $R$ s and RyRs in SMCs}

We show that between $\sim 20 \mathrm{hpf}$ to $\sim 24 \mathrm{hpf}$ there is a progressive organization of $\mathrm{IP}_{3} \mathrm{Rs}$ into distinct bands (Fig. 13 Aiii-Av). Colabeling with the F59 myosin heavy chain antibody indicates that at $\sim 24 \mathrm{hpf}$ the $\mathrm{IP}_{3}$ Rs were localized to the region of sarcoplasmic reticulum (SR) adjacent to the sarcomeric I-band, with a higher intensity of labeling in the SR adjacent to the z-line (Fig. 13 C, E). This is similar to what was found in cultured mouse myotubes, where the $\mathrm{IP}_{3} \mathrm{Rs}$ were also reported to be localized in the I-band SR (Powell et al., 2001). We were unable to detect a clear and distinctive pattern of $\mathrm{IP}_{3} \mathrm{R}$ staining in the nuclear region of SMCs in zebrafish (especially during the early SP1), as has been reported in cultured mouse myotubes (Jaimovich et al., 2000; Powell et al., 2001). This localization would have supported our calcium green-1 dextran imaging experiments with untreated embryos (Figs. 5-7) and the aequorin imaging experiments following treatment with 2-APB (Fig. 12). We tried several commercially available (as well as one custom-made zebrafish) anti-IP $R$ antibodies without success. However, we suspect that the $\mathrm{IP}_{3} \mathrm{Rs}$ responsible for generating the nuclear $\mathrm{Ca}^{2+}$ signal are most likely to be localized preferentially in the inner nuclear membrane, as has been reported in cultured rat skeletal muscle cells (Cárdenas et al., 2005). We found that this region of the nucleus is difficult to access successfully and thus label via immunohistochemistry in intact embryos, and we suggest that this might be why we do not see any $I P_{3} R$ labeling of the nuclear envelope in SMCs that would correspond to the $\mathrm{IP}_{3}$-mediated nuclear $\mathrm{Ca}^{2+}$ signaling during the early SP1.

There is also a progressive organization of RyRs into bands between $20 \mathrm{hpf}$ to $24 \mathrm{hpf}$ (Fig. $13 \mathrm{Biii}-\mathrm{Bv}$ ). Co-labeling of the RyRs and F-actin (with rhodamine-phalloidin) indicated that at 24 hpf the RyRs are also located in the l-band SR, approximately in the region of the z-line (Fig. $13 \mathrm{D}, \mathrm{F}$ ). It has been previously reported that RyRs are localized along the interface of the SR and T-tubule, at the terminal cisternae of the SR (Ward and Lederer, 2005) and that in Xenopus and zebrafish, a single T-tubule is located at the z-line (Davis and Carlson, 1994; Zhang etal., 2009), which supports our RyR immunolabeling data. In addition, Fig. $13 C$ and 13D indicate that as both $I_{3} R$ s and RyRs are localized at the $\mathrm{z}$-line $\mathrm{SR}$, both types of $\mathrm{Ca}^{2+}$ channels may be able to interact with the DHPRs located in the T-tubule either indirectly $\left(\mathrm{IP}_{3} \mathrm{Rs}\right)$ or directly (RyRs). This idea was originally proposed by Araya etal., 2003) who reported that this spatial arrangement was responsible for generating depolarization-evoked, $\mathrm{IP}_{3} \mathrm{R}$-medi- ated, slow $\mathrm{Ca}^{2+}$ signals in primary cultures of rat skeletal muscle cells. We suggest that a somewhat analogous situation may also be found in zebrafish where $\mathrm{IP}_{3} \mathrm{R}$-mediated $\mathrm{Ca}^{2+}$ release is responsible for the initial, and prolonged, signal from the nucleus, while predominantly RyR-mediated release drives the subsequent faster cytosolic phase of SMC $\mathrm{Ca}^{2+}$ signaling.

\section{Possible roles of spontaneous $\mathrm{Ca}^{2+}$ signals in slow muscle development}

To explore the possible developmental function of the spontaneous SP1 $\mathrm{Ca}^{2+}$ signals, we examined the effect of the antagonists, 2-APB and ryanodine, on the organization and localization of the key contractile proteins, actin and myosin, as well as on the overall number and gross morphology of the SMCs. Treatment with either 2-APB or ryanodine resulted in a disruption of the normal pattern of actin and myosin banding in a dose dependent fashion (Fig. $15 \mathrm{C}-\mathrm{F}$ ). Our ryanodine treatments support the observation of Brennan et al. (2005) that AChR activity generates $\mathrm{Ca}^{2+}$ signals via RyRs, and that these play a crucial role in myofibril organization. This role includes regulating both sarcomere and myofiber length, as well as the bundling and alignment of myofibrils.

Our new data show that both 2-APB and ryanodine-treated embryos have abnormal sarcomere assembly (Fig. 15). It has been previously reported, however, that treatment of tail-cut zebrafish embryos at $17 \mathrm{hpf}$ with $10 \mu \mathrm{M}$ and $50 \mu \mathrm{M}$ ryanodine leaves sarcomeric banding relatively intact (Brennan et al., 2005), whereas we report that while this is true to a certain extent for treatment with $10 \mu \mathrm{M}$ ryanodine, $50 \mu \mathrm{M}$ ryanodine treatment almost completely abolishes the banding pattern (Fig. $15 \mathrm{Fi}^{\star}, \mathrm{Fii}^{\star}$ and $\left.\mathrm{Fiii}^{\star}\right)$. This difference might be explained by the amount of the tail excised in order to expose the internal tissues to the receptor antagonists contained in the bathing medium. We attempted to remove the caudal $100 \mu \mathrm{m}$ of the tail, and found this to be sufficient to expose the anterior somite blocks to the diffusion of solutes from the bathing medium through the interior trunk intercellular space. This diffusion was assessed via tracking the spread of fluorescence through the trunk of embryos that were incubated, following the tail-cut procedure, in $\alpha$-bungarotoxin-Alexa Fluor 488.

Treatment with ryanodine did not disrupt SMC number (Fig. 16). This supports the observations previously reported by Brennan et al. (2005). On the other hand, treatment with 2-APB had a significant effect on the number of SMCs generated (Fig. 16). This observation supports our suggestion that the $\mathrm{IP}_{3} \mathrm{R}$-mediated signals may act to regulate the expression of a particular set of genes required for the differentiation of SMCs (Jaimovich et al., 2000; Powell et al., 2001; Araya et al., 2003).

Ryanodine treatment also resulted in the detachment of the SMCs from the vertical segment boundaries (especially at the dorsal and ventral regions of the somites; Fig. 15 E, F). The SMCs in the medial region of the somites tend to remain attached to the vertical segment boundaries after treatment, and could thus be seen to extend across the whole A/P length of the somite. This may result from the concentration of ryanodine being greater at the dorsal and ventral edges of the somites than in the medial regions, or that the SMCs are more mature, and thus have developed better connections with the segment boundary (Barresi et al., 2001). This result, along with the overall disruption in 
myotome shape caused by ryanodine treatment, suggests that there may be $\mathrm{Ca}^{2+}$-sensitive elements in the complex segment boundary matrix that regulate the interaction between SMCs and the boundaries. These elements may therefore play a key role in maintaining the overall architecture of the chevron-shaped myotome block during myofibrillogenesis (Henry et al., 2004). It is interesting to note that treatment with 2-APB had little effect on the overall architecture of the myotome blocks, whereas ryanodinetreatment resulted in a gross disruption, where the somites assumed a U-shaped morphology. These observations are similar to those made in cyclopamine or forskolin-treated embryos (Fig. 9) or in the smo $^{-1-}$ mutant fish (Fig. 10), when SMCs were completely absent. The data suggest that normally formed SMCs may play an important role in determining the overall morphology of the trunk myotome blocks.

Our new data thus support and extend the pioneering studies using Xenopus embryos, where $\mathrm{Ca}^{2+}$ is released predominantly from RyRs, which is intimately involved in the differentiation of skeletal muscle. This differentiation includes cytoskeletal organization during myofibrillogenesis and the formation of sarcomeres in both cultured Xenopus myocytes and maturing somites of Xenopus embryos (Ferrari et al., 1996; 1998; Ferrari and Spitzer, 1999; Campbell et al., 2006).

\section{Materials and Methods}

\section{Embryo collection}

Zebrafish (Danio rerio), including the wild-type $\mathrm{AB}$ strain, the $s m o^{+1-}$ mutant (both from the Zebrafish International Resource Centre; University of Oregon, Eugene, OR, USA) and the $\alpha$-actin-apoaequorin-IRESEGFP ( $\alpha$-actin-aeq) transgenic line, were maintained on a $14 \mathrm{hr}$ light/ 10 $\mathrm{hr}$ dark cycle to stimulate spawning (Westerfield, 1994), and their fertilized eggs were collected as described elsewhere (Webb et al., 1997). Embryos were maintained in $30 \%$ Danieau's solution $(17.4 \mathrm{mM} \mathrm{NaCl}$, $0.21 \mathrm{mM} \mathrm{KCl}, 0.18 \mathrm{mM} \mathrm{Ca}\left(\mathrm{NO}_{3}\right)_{2}, 0.12 \mathrm{mM} \mathrm{MgSO}_{4} .7 \mathrm{H}_{2} \mathrm{O}, 1.5 \mathrm{mM}$ Hepes, $\mathrm{pH} 7.2$ ) at $\sim 28.5^{\circ} \mathrm{C}$ throughout development and during all experiments.

\section{Preparation of the p $\alpha-K S$-aeq-IRES-EGFP plasmid}

To prepare the $p \alpha-K S$-aeq-IRES-EGFP plasmid (Fig. 1) used for generating the $\alpha$-actin-aeq transgenic zebrafish, PCR was carried out to amplify the apoaequorin gene from the piP-HE plasmid (Inouye et al., 1989), using the following oligonucleotide primers: 5'accagaattcatgacaagcaaacaatactcagtcaagcttacatcagac-3' and 5'accagtcgacttaggggacagctccaccgtagag-3', in which two restriction enzyme recognition sites for, ECOR1 and Sal, were added to the 5' and 3' ends of the apoaequoringene, respectively. The apoaequoringene was then cloned into the pIRES2-EGFP plasmid (Clontech Laboratories, Inc., Mountain View, CA, USA) using these restriction enzyme sites. The aeqIRES-EGFP fragment was then excised with ECOR1 and Not1, after which it was cloned into the p $\alpha$-SK plasmid (Higashijima et al., 1997) to obtain an aeq-IRES-EGFP fragment with an $\alpha$-actin promoter (i.e., $\alpha$-aeq-IRESEGFP). In parallel, the SV40 late polyadenylation signal (pA) was amplified from the $\mathrm{pCMVT}_{N}{ }^{T}$ vector (Promega Corp., Madison, WI, USA) using the following oligonucleotide primers:

5'-accagcggccgccagacatgataagatacattg-3' and

5'-accagagctctctagaaccggttaccacatttgtagaggttt-3', in which the Not recognition sequence was added to the 5' end and Age1, Xba1 and SaC1 recognition sequences were added to the 3 ' end. The SV40 late polyadenylation signal was then cloned into the pBluescriptII-KS ${ }^{+}$plasmid following Not 1 and Sac1 double digestion, after which the $\alpha$-aeq-IRES-EGFP fragment was cloned into this plasmid following Xho1 and Not double digestion. All the restriction enzymes used in this study were obtained from New England Biolabs, Inc.

\section{Generation of transgenic fish}

The $p \alpha$-KS-aeq-IRES-EGFP plasmid was linearized with $X b a 1$ and then $\sim 1 \mathrm{nl}$ (i.e., at $\sim 100-200 \mathrm{pg} / \mathrm{nl}$, prepared in nuclease-free water) was microinjected into the center of the blastodisc of zebrafish embryos at the 1-cell stage. The microinjection pipettes and pressure injection system used are described in detail elsewhere (Webb et al., 1997). The injected embryos were raised to adulthood and were then intercrossed with wildtype $A B$ fish. The expression of EGFP in the offspring was used to identify the $\alpha$-actin-aeq transgenic germ-line founder fish and these were subsequently used to establish the stable homozygous transgenic line. EGFP fluorescence was visualized with a Nikon D-Eclipse $C 1$ scanning confocal system using a Nikon Fluor 10x/0.3NA objective lens, a $488 \mathrm{~nm}$ excitation laser and a 515/530 nm emission filter.

\section{In vivo reconstitution of aequorin and aequorin-based $\mathrm{Ca}^{2+}$ imaging}

The $\alpha$-actin-aeq transgenic embryos (and, for some experiments, aeqmRNA injected embryos) were dechorionated manually with watchmaker's forceps at the 8-cell stage and then incubated in a custom-designed holding/imaging chamber with $50 \mu \mathrm{M} f$-coelenterazine (Molecular Probes, Invitrogen Corp., Carlsbad, CA, USA) in 30\% Danieau's solution to reconstitute the active aequorin. Apoaequorin was transiently expressed in zebrafish embryos by injecting $\sim 1 \mathrm{nl}$ (i.e., at $\sim 500 \mathrm{pg} / \mathrm{hl}$, prepared in nuclease-free water) aeq-mRNA into the center of the yolk at the 1-cell stage following protocols described by Cheung et al. (2006). The $f$ coelenterazine was prepared as a stock solution of $5 \mathrm{mM}$ in $100 \%$ methanol and was diluted in 30\% Danieau's solution just prior to use. Chambers containing embryos bathed in $f$-coelenterazine solution were then transferred to a custom-built Photon Imaging Microscope System (PIMS; Science Wares, East Falmouth, MA, USA) for data acquisition from $\sim 16.5$ to $24 \mathrm{hpf}$ (i.e., the 15 - to 30 -somite stage) at $\sim 28.5^{\circ} \mathrm{C}$. An outline of the PIMS and the photon acquisition procedure is described by Webb et al. (1997). The imaging software, IpdWin2k, allowed the collected photon data to be subsequently analyzed with any chosen integration time and region of interest (ROI). All data were exported to Microsoft Office Excel 2003 (Microsoft Corp., Redmond, WA, USA), GraphPad Prism 5 (GraphPad Software, Inc., San Diego, CA, USA) and Corel Draw X3 (Corel Corp., Ottawa, Canada) for statistical analysis and figure preparation.

\section{Western blot analysis}

Embryos of the $\alpha$-actin-aeq transgenic line were collected at 12, 14, $18,20,22$ and $24 \mathrm{hpf}$ for the preparation of protein extracts used in Western blot analysis. The preparation of protein extracts and Western blot analysis were conducted as described by Cheung et al. (2006). Extract prepared from 3 embryos was loaded into each lane of the SDSpolyacrylamide gel. 20 pg AquaLite ${ }^{\circledR}$ aequorin (Molecular Probes) was used as an internal control and the extract from wild-type embryos at 24 hpf was used as a negative control.

\section{Calcium green-1 dextran imaging}

Wild-type embryos were microinjected at the 1-cell stage with $2 \mathrm{nl}$ of a mixture of the fluorescent $\mathrm{Ca}^{2+}$-sensitive indicator, calcium green-1 dextran (10S; $200 \mu \mathrm{M}$; Molecular Probes), and the $\mathrm{Ca}^{2+}$-insensitive fluorescent dye, rhodamine B dextran (10S; $200 \mu \mathrm{M}$; Sigma-Aldrich Corp). The injected embryos were then maintained at $\sim 28.5^{\circ} \mathrm{C}$ until the start of the Segmentation Period. A series of time-lapse images was then recorded in somites $7-9$ of the injected embryos at $\sim 17.5 \mathrm{hpf}$ (i.e., the 17somite stage) and $\sim 24 \mathrm{hpf}$ (i.e., prim-5 stage) using the Nikon D-Eclipse C1 scanning confocal system, described previously, with a Nikon Fluor 40x/0.8NA water dipping objective lens. Calcium green-1 dextran-generated fluorescence was acquired using a $488 \mathrm{~nm}$ excitation laser and a $515 / 530 \mathrm{~nm}$ emission filter while rhodamine dextran-generated fluorescence was acquired using a $543 \mathrm{~nm}$ excitation laser and a $570 \mathrm{~nm}$ (long pass) emission filter. In addition, a series of time-lapse images was recorded in somites $7-10$ of injected embryos at $\sim 18.5 \mathrm{hpf}$ (i.e., the 19- 
somite stage) using a Nikon D-Eclipse C1si scanning confocal system mounted on a Nikon Eclipse 80i upright microscope with a Nikon Achromat $40 x / 0.8 N A$ water dipping objective lens. With this system, green fluorescence was acquired using a $488 \mathrm{~nm}$ excitation laser and a 525/550 $\mathrm{nm}$ (band pass) emission filter, and red fluorescence was acquired using a $561 \mathrm{~nm}$ excitation laser and a $605 / 660 \mathrm{~nm}$ (band pass) emission filter. To achieve a significant period of uninterrupted fluorescence-based $\mathrm{Ca}^{2+}$ imaging, 160 images were acquired over a period of $100 \mathrm{sec}$, first with $488 \mathrm{~nm}$ excitation (for $\mathrm{Ca}^{2+}$ imaging), and then with $543 \mathrm{~nm}$ (Eclipse C1) or $561 \mathrm{~nm}$ (Eclipse $\mathrm{C} 1 \mathrm{si}$ ) excitation (to monitor $\mathrm{Ca}^{2+}$-independent changes in fluorescence). All these data were acquired via the Nikon EZ-C1 3.40 software. This software was also used to review and analyze the calcium green-1 dextran and rhodamine dextran-generated fluorescence. At $\sim 17.5 \mathrm{hpf}$ and $\sim 24 \mathrm{hpf}$, the average fluorescence intensity of a circular sampling ROI covering $\sim 4-5$ SMCs in somite 8 was acquired. At $\sim 18.5 \mathrm{hpf}$, this same ROI was placed in the middle of somites $7,8,9$ and 10 to compare the $\mathrm{Ca}^{2+}$ signals generated in adjoining somites, while for the comparison of $\mathrm{Ca}^{2+}$ signals generated in different regions of the same somite, the ROls were placed in the dorsal, middle and ventral regions of somite 9 .

To analyze the $\mathrm{Ca}^{2+}$ signals generated from a single muscle cell located in somite 10, the total fluorescence intensities in the nucleus alone and in the entire muscle cell were measured, and then the total fluorescence intensity in the cytoplasm was determined by subtracting the total fluorescence intensity of nucleus from the total fluorescence intensity of the entire muscle cell. The fold-change in fluorescence intensity was then calculated by determining the ratio of the maximum fluorescence intensity in relation to the basal level.

All data were exported to Microsoft Office Excel 2000, GraphPad Prism 5 and Corel Draw X3 for figure preparation and statistical analysis.

\section{Inhibition of slow muscle development using a pharmacological approach: cyclopamine and forskolin treatment}

To block the development of SMCs, wild-type embryos were incubated either in $0.1 \mathrm{mM}$ cyclopamine (Calbiochem, EMD Chemical, Inc., Gibbstown, NJ, USA; Barresi et al., 2000; Hirsinger et al., 2004) dissolved in 1\% DMSO or in $0.15 \mathrm{mM}$ forskolin (Calbiochem; Barresi et al., 2000) dissolved in 2\% DMSO in 30\% Danieau's solution, from $5.5 \mathrm{hpf}$ to $\sim 24$ hpf. As a control, some embryos were incubated in $2 \%$ DMSO in $30 \%$ Danieau's solution alone. Bright-field images of these embryos were captured at $\sim 24 \mathrm{hpf}$ using a Nikon DXM 1200F camera mounted on a Zeiss Axioskop microscope with a Zeiss Plan Neofluar 10x/0.3NA objective lens. Fluorescence labeling was then conducted using the F59 myosin heavy chain antibody to confirm the loss of SMCs in the cyclopamine- and forskolin- treated embryos.

In another series of experiments, the $\alpha$-actin-aeq transgenic embryos were initially incubated in $f$-coelenterazine solution to reconstitute active aequorin in vivo at the 8-cell stage and then were incubated in either 0.1 $\mathrm{mM}$ cyclopamine or $0.15 \mathrm{mM}$ forskolin in $f$ coelenterazine solution starting at $~ 5.5 \mathrm{hpf}$. These cyclopamine- and forskolin-treated transgenic embryos were then imaged using the PIMS from $\sim 17 \mathrm{hpf}$ to $\sim 21 \mathrm{hpf}$.

\section{Inhibition of slow muscle development using a genetic approach: the smo-/- mutant}

The slow-muscle-omitted (smo; also known as $s m u$ ) mutant contains mutations in the smogene that leads to many developmental processes involving hedgehog signaling being disrupted (e.g. slow muscle formation: Barresi et al., 2000). In this study, a recessive lethal mutant line, $s m o^{b 557 / b 557}$, was used. For the $\mathrm{Ca}^{2+}$ imaging experiments, normal control (i.e., $s m o^{+/+}$or $s m o^{+/}$) and $s m o^{-/}$embryos were initially injected with aeqmRNA at the 1-cell stage and then were incubated in f-coelenterazine solution to reconstitute active aequorin in vivo at the $\sim 8$-cell stage. These injected embryos were imaged using the PIMS from $\sim 17 \mathrm{hpf}$ to $\sim 21 \mathrm{hpf}$. As aequorin is expressed throughout the entire embryo in aeq-mRNAinjected embryos, the average luminescent intensity of a circular sam- pling ROI covering $\sim 8000 \mu \mathrm{m}^{2}$ on somite 8 was plotted over time. This served to eliminate the possibility of collecting aequorin-generated light from other parts of the embryo.

\section{Blocking $\mathrm{Ca}^{2+}$ signals using a pharmacological approach}

The $\alpha$-actin-aeq transgenic embryos were incubated in $f$ coelenterazine solution to reconstitute active aequorin in vivo. These embryos were imaged using the PIMS and the FB15 luminometer (Zylux Corp., Maryville, $\mathrm{TN}, \mathrm{USA}$ ) at $\sim 17 \mathrm{hpf}$ and then they were treated with either $25 \mu \mathrm{M} 2-\mathrm{APB}$ (Sigma-Aldrich Corp) or $50 \mu \mathrm{M}$ ryanodine (Calbiochem) for $3 \mathrm{hrs}$ starting at $\sim 17.5 \mathrm{hpf}$ (i.e., when the $\mathrm{Ca}^{2+}$ signals started) to block $\mathrm{Ca}^{2+}$ release from $I_{3}$ Rs or RyRs, respectively. The terminal $\sim 100 \mu \mathrm{m}$ region of the tip of the tail bud was excised (i.e., such that the posterior-most, newly formed somites were not damaged) using a tungsten needle $(27 \mathrm{G} 1 / 2$ Becton Dickinson \& Co., Franklin Lakes, NJ, USA) just prior to the $\mathrm{Ca}^{2+}$ channel antagonist treatments, to ensure that the drugs diffused into the trunk tissues of the later-stage embryos (Liu and Westerfield, 1990). In another series of experiments, tail-tip excised wild-type embryos were treated from $\sim 17 \mathrm{hpf}$ to $20 \mathrm{hpf}$ with 2 -APB (at $25 \mu \mathrm{M}$ or $50 \mu \mathrm{M}$ ) or with ryanodine (at $10 \mu \mathrm{M}$ or $50 \mu \mathrm{M}$ ) after which they were fixed immediately prior to dual labeling of myosin and actin.

\section{$\alpha$-Bungarotoxin and nifedipine treatment}

The $\alpha$-actin-aeq transgenic embryos were incubated in $f$ coelenterazine solution to reconstitute active aequorin in vivo. The tip of the tail was excised as described previously, after which embryos were treated with either $3 \mu \mathrm{M} \alpha$-bungarotoxin-Alexa Fluor 488 (Molecular Probes) or 500 $\mu \mathrm{M}$ nifedipine (Sigma-Aldrich Corp.) in 30\% Danieau's solution at $~ 16.5$ hpf. The former is an inhibitor of nicotinic acetylcholine receptors, nAChRs (Wonnacott, 1986; Pugh and Berg, 1994), while the latter is an organic blocker of the L-type voltage sensitive $\mathrm{Ca}^{2+}$ channel, which targets the dihydropyridine binding site on the $\alpha$-subunit of the L-type $\mathrm{Ca}^{2+}$ channel (Zamponi, 1997; Fischer and Schäfer, 2002). The Alexa Fluor 488 conjugate of $\alpha$-bungarotoxin was used so that the distribution of the toxin could be determined within the musculature of the embryonic trunk. The luminescence of these embryos was then detected using the FB15 luminometer from $\sim 17$ to $\sim 21 \mathrm{hpf}$. Triton X-100 was added to "burn-out" all remaining aequorin at the end of the experiment.

\section{Immunohistochemistry and rhodamine phalloidin labeling}

The F59 lgG1 myosin heavy chain antibody (Crow and Stockdale, 1986) was used to label the slow muscle in the trunk of zebrafish embryos from $\sim 16 \mathrm{hpf}$ to $\sim 24 \mathrm{hpf}$. Tissue culture supernatant of the F59 antibody (Developmental Studies Hybridoma Bank, lowa City, IA, USA) was used at a dilution of $1: 10$. The $34 \mathrm{C}$ monoclonal anti-ryanodine receptor antibody (Sigma-Aldrich Corp., MO, USA; Brennan et al., 2005) recognizes RyR-1 and RyR-2, and was used at a dilution of 1:500. The anti-InsP3R, Type 1 polyclonal IP ${ }_{3} R$ antibody (Upstate, Millipore Corp., Temecula, CA, USA) recognizes the type $1 \mathrm{IP}_{3} R$ and was also used at a dilution of 1:500. Rhodamine phalloidin (Molecular Probes), which labels F-actin, was used at a dilution of 1:200 in most experiments. However, when conducting dual labeling, it was used at a dilution of 1:50.

Embryos were dechorionated manually using watchmaker's forceps and then fixed with $4 \%$ paraformaldehyde in PBS ( $\mathrm{pH} \mathrm{7.3;} \mathrm{Westerfield}$ 1994) overnight at $4^{\circ} \mathrm{C}$, after which they were washed thoroughly with PBS. Embryos were washed with PBS containing $0.1 \%$ triton X-100 twice for 5 min and once with PBS containing $0.1 \%$ triton $X-100$ and $1 \%$ DMSO (PBTD) for $5 \mathrm{~min}$. For labeling with the F59 or 34C antibodies, embryos were then incubated with blocking buffer (PBTD containing $10 \%$ goat serum and $1 \% \mathrm{BSA}$ ) while for labeling with the anti-InsP3R, embryos were initially blocked in PBTD containing 10\% BSA at room temperature for 2 hrs. Embryos were then incubated with the appropriate primary antibody overnight at $4^{\circ} \mathrm{C}$ in the dark, after which they were washed extensively with PBTD containing 1\% BSA (PBTD/BSA). For the F59 and 34C labeling, a FITC-conjugated goat anti-mouse secondary antibody (stock 
solution of $1.5 \mathrm{mg} / \mathrm{ml}$; Jackson Immuno Research Laboratories, Inc., PA, USA), was used, while for the anti-InsP3R labeling, an Alexa Fluor 568 goat anti-rabbit secondary antibody (stock solution of $2 \mathrm{mg} / \mathrm{ml}$; Molecular Probes, Invitrogen Corp., Carlsbad, CA, USA) was used. Both of these secondary antibodies were prepared at a dilution of 1:200 in blocking buffer just prior to use. The embryos were incubated with the secondary antibody overnight at $4^{\circ} \mathrm{C}$ in the dark and they were then washed extensively with PBTD/BSA.

The fluorescence images of labeled embryos were acquired with the Nikon D-Eclipse C1 scanning confocal system described previously using a Nikon Fluor 60x/1.0NA water-dipping objective lens. Green fluorescence was captured using a $488 \mathrm{~nm}$ excitation wavelength and a 515/530 $\mathrm{nm}$ emission filter, while red fluorescence was captured using a $543 \mathrm{~nm}$ excitation wavelength and $570 \mathrm{~nm}$ (long pass) emission filter.

\section{Acknowledgements}

We would like to thank Anming Meng (Tsinghua University, Beijing, PRC) and Frederick I. Tsuji (Scripps Institution of Oceanography, University of California San Diego, USA) for providing us with the $\alpha p$-SK and piPHE plasmids, respectively. We would also like to thank Robert Baker (NYUMedical School, New York, USA) for his helpful advice with regards to the developmental fates of cells derived from anterior somites. This work was funded by RGC grants HKUST6416/06M, HKUST661707 and HKUST662109 awarded to A.L.M. The zebrafish were supplied by the Zebrafish International Resource Center, supported by grant \#RR12546 from the NIH-NCRR.

\section{References}

ARAYA, R., LIBERONA, J.L., CARDENAS, J.C., RIVEROS, N., ESTRADA, M., POWELL, J.A., CARRASCO, M.A. and JAIMOVICH, E. (2003). Dihydropyridine receptors as voltage sensors for a depolarization-evoked, $I P_{3} R$-mediated, slow calcium signal in skeletal muscle cells. J Gen Physio/121: 3-16.

ASHWORTH, R. and BOLSOVER, S.R. (2002). Spontaneous activity-independent intracellular calcium signals in the developing spinal cord of the zebrafish embryo. Dev Brain Res 139: 131-137.

ARRESI, M.J., STICKNEY, H.L. and DEVOTO, S.H. (2000). The zebrafish slowmuscle-omitted gene product is required for Hedgehog signal transduction and the development of slow muscle identity. Development 127: 2189-2199.

BARRESI, M.J., D'ANGELO, J.A., HERNÁNDEZ, L.P. and DEVOTO, S.H. (2001). Distinct mechanisms regulate slow-muscle development. Curr Bio/11: 14321438.

BLAGDEN, C.S., CURRIE, P.D., INGHAM, P.W. and HUGHES, S.M. (1997). Notochord induction of zebrafish slow muscle mediated by Sonic hedgehog. Genes Dev 11: 2163-2175.

BRENNAN, C., AMACHER, S.L. and CURRIE, P.D. (2002). IV. Aspects of organogenesis: Somitogenesis. Res Probl Cell Differ 40: 271-297.

BRENNAN, C., MANGOLI, M., DYER, C.E.F. and ASHWORTH, R. (2005). Acetylcholine and calcium signaling regulates muscle fibre formation in the zebrafish embryo. J Cel/ Sci118: 5181-5190.

CAMPBELL, N.R., PODUGU, S.P. and FERRARI, M.B. (2006). Spatiotemporal characterization of short versus long duration calcium transients in embryonic muscle and their role in myofibrillogenesis. Dev Bio/292: 253-264.

CÁRDENAS, C., LIBERONA, J.L., MOLGÓ, J., COLASANTE, C., MIGNERY, G.A. and JAIMOVICH, E. (2005). Nuclear inositol 1,4,5-trisphosphate receptors regulate local $\mathrm{Ca}^{2+}$ transients and modulate cAMP response element binding protein phosphorylation. J Cell Sci118: 3131-3140.

CARRASCO, M.A., RIVEROS, N., RÍOS, J., MÜLLER, M., TORRES, F., PINEDA, J., LANTADILLA, S. and JAIMOVICH, E. (2003). Depolarization-induced slow calcium transients activate early genes in skeletal muscle cells. Am J Physiol Cell Physio/284: C1438-C1447.

CHEN, J.K., TAIPALE, J., COOPER, M.K. and BEACHY, P.A. (2002). Inhibition of Hedgehog signaling by direct binding of cyclopamine to Smoothened. Genes Dev 16: 2743-2748.

CHEUNG, C.Y., WEBB, S.E., MENG, A. and MILLER, A.L. (2006). Transient expression of apoaequorin in zebrafish embryos: extending the ability to image calcium transients during later stages of development. Int J Dev Bio/50: 561 . 569.

CRÉTON, R., SPEKSNIJDER, J.E. and JAFFE, L.F. (1998). Patterns of free calcium in zebrafish embryos. J Cell Sci111: 1613-1622.

CROW, M.T. and STOCKDALE, F.E. (1986). Myosin expression and specialization among the earliest muscle fibers of the developing avian limb. Dev Bio/113: 238-254.

CURRIE, P.D. and INGHAM, P.W. (1996). Induction of a specific muscle cell type by a hedgehog-like protein in zebrafish. Nature 382: 452-455.

DAVID, J.D., SEE, W.M. and HIGGINBOTHAM, C.A. (1981). Fusion of chick embryo skeletal myoblasts: Role of calcium influx preceding membrane union. Dev Bio/82: 297-307.

DAVIS, A.K. and CARLSON, S.S. (1994). Proteoglycans are present in the transverse tubule system of skeletal muscle. Matrix Bio/14: 607-621.

DEVOTO, S.H., MELANCON, E., EISEN, J.S. and WESTERFIELD, M. (1996). Identification of separate slow and fast muscle precursor cells in vivo, prior to somite formation. Development 122: 3371-3780.

DRAPEAU, P., SAINT-AMANT, L., BUSS, R.R., CHONG, M., MCDEARMID, J.R and BRUSTEIN, E. (2002). Development of the locomotor network in zebrafish. Prog Neurobio/68: 85-111.

DU, S.J., DEVOTO, S.H., WESTERFIELD, M. and MOON, R.T. (1997). Positive and negative regulation of muscle cell identity by members of the hedgehog and TGF- $\beta$ gene families. J Cel/ Bio/139: 145-156.

EISEN, J.S., MYERS, P.Z. and WESTERFIELD, M. (1986). Pathway selection by growth cones of identified motoneurones in live zebra fish embryos. Nature 320 269-271

FAHRENKRUG, S.C., CLARK, K.J., DAHLQUIST, M.O. and HACKETT, P.B. JR. (1999). Dicistronic gene expression in developing zebrafish. Mar Biotechnol. (NY) 1: 552-561.

FERRARI, M.B., ROHRBOUGH, J. and SPITZER, N.C. (1996). Spontaneous calcium transients regulate myofibrillogenesis in embryonic Xenopus myocytes. Dev Bio/178: 484-497.

FERRARI, M.B., RIBBECK, K., HAGLER, D.J. and SPITZER, N.C. (1998). A calcium signaling cascade essential for myosin thick filament assembly in Xenopus myocytes. J Cel/ Bio/141: 1349-1356.

FERRARI, M.B. and SPITZER, N.C. (1999). Calcium signaling in the developing Xenopus myotome. Dev Bio/213: 269-282.

FISCHER, M. and SCHÄFER, S.S. (2002). Effects of the calcium antagonist nifedipine on the afferent impulse activity of isolated cat muscle spindles. Brain Res 954: 256-276.

FUJITA, H., NEDACHI, T. and KANZAKI, M. (2007). Accelerated de novo sarcomere assembly by electric pulse stimulation in $\mathrm{C} 2 \mathrm{C} 12$ myotubes. Exp Cell Res 313: 1853-1865.

GILLAND, E., MILLER, A.L., KARPLUS, E., BAKER, R. and WEBB, S.E. (1999). Imaging of multicellular large-scale rhythmic waves during zebrafish gastrulation. Proc Natl Acad Sci USA 96: 157-161.

GRUNWALD, D.J., KIMMEL, C.B., WESTERFIELD, M., WALKER, C. and STREISINGER, G. (1988). A neural degeneration mutation that spares primary neurons in the zebrafish. Dev Bio/126: 115-128.

HALPERN, M.E., HO, R.K., WALKER, C. and KIMMEL, C.B. (1993). Induction of muscle pioneers and floor plate is distinguished by the zebrafish no tail mutation. Cell 75: 99-111.

HENRY, C.A. and AMACHER, S.L. (2004). Zebrafish slow muscle cell migration induces a wave of fast muscle morphogenesis. Dev Cell 7: 917-923.

HIGASHIJIMA, S., OKAMOTO, H., UENO, N., HOTTA, Y. and EGUCHI, G. (1997). High-frequency generation of transgenic zebrafish which reliably express GFP in whole muscles or the whole body by using promoters of zebrafish origin. Dev Bio/192: 289-299.

HIRSINGER, E., STELLABOTTE, F., DEVOTO, S.H. and WESTERFIELD, M (2004). Hedgehog signaling is required for commitment but not initial induction of slow muscle precursors. Dev Bio/275: 143-157.

HOLLEY, S.A. and NÜSSLEIN-VOLHARD, C. (2000). Somitogenesis in zebrafish. Curr Top Dev Biol 47: 247-277.

HOLLEY, S.A. (2006). Anterior-posterior differences in vertebrate segments: speci- 
fication of trunk and tail somites in the zebrafish blastula. Genes Dev 20: 1831 1837.

INOUYE, S., AOYAMA, S., MIYATA, T., TSUJI, F.I. and SAKAKI, Y. (1989). Overexpression and purification of the recombinant $\mathrm{Ca}^{2+}$-binding protein, apoaequorin. J Biochem (Tokyo) 105: 473-477.

JAIMOVICH, E., REYES, R., LIBERONA, J.L. and POWELL, J.A. (2000). IP receptors, $\mathrm{IP}_{3}$ transients, and nucleus-associated $\mathrm{Ca}^{2+}$ signals in cultured skeletal muscle. Am J Physiol Cell Physio/278: C998-C1010.

JAIMOVICH, E. and CARRASCO, M.A. (2002). IP ${ }_{3}$ dependent $\mathrm{Ca}^{2+}$ signals in muscle cells are involved in regulation of gene expression. Biol Res 35: 195202.

JANG, S.K., KRAUSSLICH, H.G., NICKLIN, M.J., DUKE, G.M., PALMENBERG, A.C. and WIMMER, E. (1988). A segment of the 5' nontranslated region of encephalomyocarditis virus RNA directs internal entry of ribosomes during in vitro translation. J Viro/62: 2636-2643.

KIMMEL, C.B., KANE, D.A., WALKER, C., WARGA, R.M. and ROTHMAN, M.B. (1989). A mutation that changes cell movement and cell fate in the zebrafish embryo. Nature 337: 358-362.

KIMMEL, C.B., WARGA, R.M. and SCHILLING, T.F. (1990). Origin and organization of the zebrafish fate map. Development 108: 581-594.

LECLERC, C., WEBB, S.E., DAGUZAN, C., MOREAU, M. and MILLER, A.L. (2000). Imaging patterns of calcium transients during neural induction in Xenopus laevis embryos. J Cell Sci113: 3519-3529.

LECLERC, C., LEE, M., WEBB, S.E., MOREAU, M. and MILLER, A.L. (2003). Calcium transients triggered by planar signals induce the expression of $Z / C 3$ gene during neural induction in Xenopus. Dev Bio/261: 381-390.

LEUNG, C.F., MILLER, A.L., KORZH, V. and WEBB, S.E. (2009). Visualization of stochastic $\mathrm{Ca}^{2+}$ signals in the formed somites during the early Segmentation Period in intact, normally developing zebrafish embryos. Dev Growth Differ 51 : 617-637.

LI, H., COOK, J.D., TERRY, M., SPITZER, N.C. and FERRARI, M.B. (2004). Calcium transients regulate patterned actin assembly during myofibrillogenesis. Dev Dyn 229: 231-242.

LIU, D.W. and WESTERFIELD, M. (1990). The formation of terminal fields in the absence of competitive interactions among primary mononeurons in the zebrafish. $J$ Neurosci 10, 3947-3959.

LIU, D.W. and WESTERFIELD, M. (1992). Clustering of muscle acetylcholine receptors requires motoneurons in live embryos, but not in cell culture. $J$ Neurosci12: 1859-1866.

LORENZON, P., GIOVANNELLI, A., RAGOZZINO, D., EUSEBI, F. and RUZZIER, F. (1997). Spontaneous and repetitive calcium transients in C2C12 mouse myotubes during in vitro myogenesis. Eur J Neuro 9: 800-808.

MA, L.H., PUNNAMOOTTIL, B., RINKWITZ, S. and BAKER, R. (2009). Mosaic hoxb4a neuronal pleiotropism in zebrafish caudal hindbrain. PLOS ONE 4(6): e5944.

MELANÇON, E., LIU, D.W., WESTERFIELD, M. and EISEN, J.S. (1997). Pathfinding by identified zebrafish motoneurons in the absence of muscle pioneers. $J$ Neurosci 17: 7796-7804

MYERS, P.Z., EISEN, J.S. and WESTERFIELD, M. (1986). Development and axonal outgrowth of identified motoneurons in the zebrafish. J Neurosci 6 :
2278-2289.

$\mathrm{OCHI}, \mathrm{H}$. and WESTERFIELD, M. (2007). Signaling networks that regulate muscle development: Lessons from zebrafish. Develop Growth Differ 49: 1-11.

POWELL, J.A., CARRASCO, M.A., ADAMS, D.S., DROUET, B., RIOS, J., MULLER, M., ESTRADA, M. and JAIMOVICH, E. (2001). IP ${ }_{3}$ receptor function and localization in myotubes: an unexplored $\mathrm{Ca}^{2+}$ signaling pathway in skeletal muscle. J Cel/ Sci114: 3673-3683.

PUGH, P.C. and BERG, D.K. (1994). Neuronal acetylcholine receptors that bind $\alpha-$ bungarotoxin mediate neurite retraction in a calcium-dependent manner. $J$ Neurosci 14: 889-896.

SAINT-AMANT, L. and DRAPEAU, P. (1998). Time course of the development of motor behaviors in the zebrafish embryo. JNeurobio/37: 622-632.

SCHNEIDER, M.F. and CHANDLER, W.K. (1973). Voltage dependent charge movement of skeletal muscle: a possible step in excitation-contraction coupling. Nature 242: 244-246.

STICKNEY, H.L., BARRESI, M.J. and DEVOTO, S.H. (2000). Somite development in zebrafish. Dev Dyn 219: 287-303.

SZETO, D.P. and KIMELMAN, D. (2006). The regulation of mesodermal progenitor cell commitment to somitogenesis subdivides the zebrafish body musculature into distinct domains. Genes Dev20: 1923-1932.

WARD, C.W. and LEDERER, W.J. (2005). Ghost sparks. Nat Cel/ Biol 7: 457-459.

WANG, X., WAN, H., KORZH, V. and GONG, Z. (2000). Use of an IRES bicistronic construct to trace expression of exogenously introduced mRNA in zebrafish embryos. Biotech 29: 814-816, 818,820.

WEBB, S.E., LEE, K.W., KARPLUS, E. and MILLER, A.L. (1997). Localized calcium transients accompany furrow positioning, propagation, and deepening during the early cleavage period of zebrafish embryos. Dev Bio/192: 78-92.

WEBB, S.E. and MILLER, A.L. (2000). Calcium signaling during zebrafish embryonic development. BioEssays 22: 113-123.

WEINBERG, E.S., ALLENDE, M.L., KELLY, C.S., ABDELHAMID, A., MURAKAMI T., ANDERMANN, P., DOERRE, O.G., GRUNWALD, D.J. and RIGGLEMAN, B. (1996). Developmental regulation of zebrafish MyoD in wild-type, no tail and spadetailembryos. Development 122: 271-280.

WESTERFIELD, M. (1994). The Zebrafish Book: A Guide for the Laboratory Use of Zebrafish (Brachydanio rerio). Univ. of Oregon Press, Eugene, OR.

WESTERFIELD, M., MCMURRAY, J.V. and EISEN, J.S. (1986). Identified motoneurons and their innervation of axial muscles in the zebrafish. JNeurosci 6 : 2267-2277.

WOLFF, C., ROY, S. and INGHAM, P.W. (2003). Multiple muscle cell identities induced by distinct levels and timing of hedgehog activity in the zebrafish embryo. Curr Bio/13: 1169-1181.

WONNACOTT, S. (1986). $\alpha$-Bungarotoxin binds to low-affinity nicotine binding sites in rat brain. $J$ Neurochem 47: 1706-1712.

ZAMPONI, G.W. (1997). Antagonist sites of voltage-dependent calcium channels. Drug Dev Res 42:131-143.

ZHANG, R., YANG, J., ZHU, J. and XU, X. (2009). Depletion of zebrafish Tcap leads to muscular dystrophy via disrupting sarcomere-membrane interaction, not sarcomere assembly. Hum Mol Genet 18: 4130-4140. 


\section{Further Related Reading, published previously in the Int. J. Dev. Biol.}

See our recent Special Issue Developmental Hematopoiesis

edited by Charles Durand, Tierry Jaffredo and Alexander Medvinsky at:

http://www.ijdb.ehu.es/web/contents.php?vol=54\&issue=6-7

Distinct patterns of MMP-9 and MMP-2 activity in slow and fast twitch skeletal muscle regeneration in vivo Malgorzata Zimowska, Edyta Brzoska, Marta Swierczynska, Wladyslawa Streminska and Jerzy Moraczewski Int. J. Dev. Biol. (2008) 52: 307-314

Regionalized calcium signaling in zebrafish fertilization

Dipika Sharma and William H. Kinsey

Int. J. Dev. Biol. (2008) 52: 561-570

Transient expression of apoaequorin in zebrafish embryos: extending the ability to image calcium transients during later stages of development

Chris Y. Cheung, Sarah E. Webb, Anming Meng and Andrew L. Miller

Int. J. Dev. Biol. (2006) 50: 561-569

Ca2+-independent protein kinase $\mathbf{C}$ signalling in mouse eggs during the early phases of fertilization Carla Tatone, Simona Delle Monache, Antonella Francione, Luisa Gioia, Barbara Barboni and Rosella Colonna Int. J. Dev. Biol. (2003) 47: 327-333

The redistribution of $\mathrm{Ca} 2+$ stores with inositol 1,4,5-trisphosphate receptor to the cleavage furrow in a microtubule-dependent manner

F Mitsuyama and T Sawai

Int. J. Dev. Biol. (2001) 45: 861-868

Restricted expression of the zebrafish hsp90alpha gene in slow and fast muscle fiber lineages

J B Sass, C C Martin and P H Krone

Int. J. Dev. Biol. (1999) 43: 835-838

Fetal myoblast clones contribute to both fast and slow fibres in developing rat muscle

$S$ M Hughes

Int. J. Dev. Biol. (1999) 43: 149-155

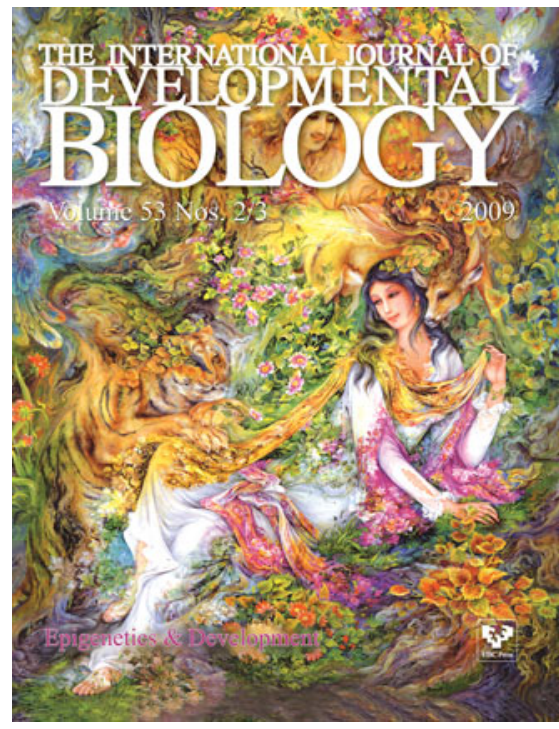

5 yr ISI Impact Factor $(2009)=3.253$

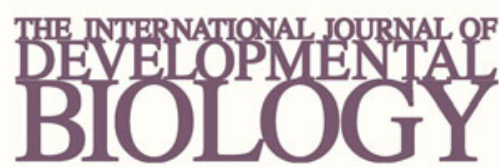

Volume 54 Nos. 6/7
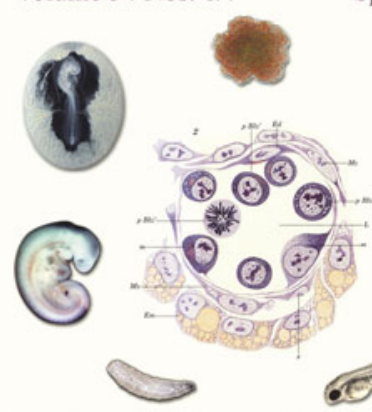

Developmental Hematopoiesis
Special Issue
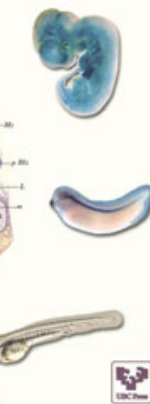

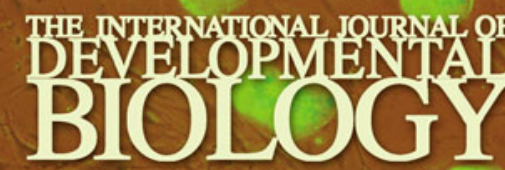

Volume 54 Nos. 11/12

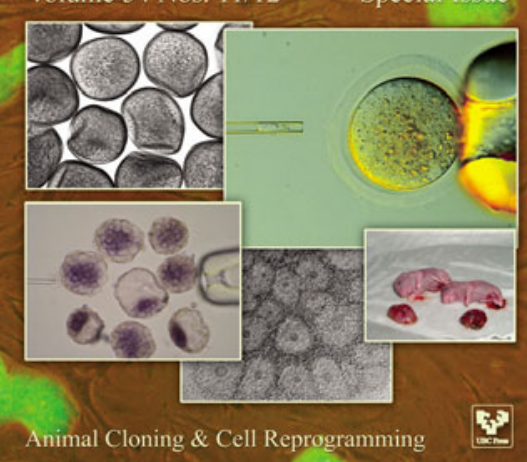

